\title{
Estimation of river pollution source using the space-time radial basis collocation method
}

\author{
Zi Li ${ }^{\mathrm{a}, \mathrm{b}}$, Xian-Zhong Mao a,*, Tak Sing Lic ${ }^{\mathrm{c}}$, Shiyan Zhang ${ }^{\mathrm{d}}$ \\ a Division of Ocean Science and Technology, Graduate School at Shenzhen, Tsinghua University, Shenzhen 518055, China \\ ${ }^{\mathrm{b}}$ School of Civil Engineering, University of Queensland, Brisbane QLD 4072, Australia \\ ' Open University of Hong Kong, Hong Kong, China \\ d Key Laboratory of Water Cycle and Related Land Surface Processes, Institute of Geographic Sciences and Natural Resources Research, Chinese Academy of \\ Sciences, Beijing 100101, China
}

\section{A R T I C L E I N F O}

\section{Article history:}

Received 19 May 2015

Revised 24 November 2015

Accepted 24 November 2015

Available online 17 December 2015

\section{Keywords:}

Contaminant source identification

Global space-time collocation model

K-fold cross-validation

Inverse problem

Radial basis function

\begin{abstract}
A B S T R A C T
River contaminant source identification problems can be formulated as an inverse model to estimate the missing source release history from the observed contaminant plume. In this study, the identification of pollution sources in rivers, where strong advection is dominant, is solved by the global space-time radial basis collocation method (RBCM). To search for the optimal shape parameter and scaling factor which strongly determine the accuracy of the RBCM method, a new cost function based on the residual errors of not only the observed data but also the specified governing equation, the initial and boundary conditions, was constructed for the $k$-fold cross-validation technique. The performance of three global radial basis functions, Hardy's multiquadric, inverse multiquadric and Gaussian, were also compared in the test cases. The numerical results illustrate that the new cost function is a good indicator to search for near-optimal solutions. Application to a real polluted river shows that the source release history is reasonably recovered, demonstrating that the RBCM with the $k$-fold cross-validation is a powerful tool for source identification problems in advectiondominated rivers.
\end{abstract}

(c) 2015 Elsevier Ltd. All rights reserved.

\section{Introduction}

Rivers and streams are quite vulnerable to pollution because they are naturally open, easily accessible and substantially used in agricultural, industrial and municipal processes. The release of hazardous contaminants into rivers, for example, sewage treatment plant emissions, accidental spills or intentional dumping of toxic materials, poses great threats to the residents and ecosystems exposed to the river water. According to the China Statistical Yearbook [16], a total of 9339 water pollution accidents took place in China from 1997 to 2008 , some of which triggered serious consequences. For example, an explosion occurred in a benzene plant maintained by a Petrochemical Company in Jilin Province, China in November 2005, resulting in approximately 100 tons of benzene being spilled into Songhuajiang River with the fire-fighting water causing severe ecological problems downstream, and millions of residents living downstream suffered from a shortage of water supply for up to four days. This incident demonstrates that it is essential to develop reliable techniques to identify the missing contaminant source information in water

\footnotetext{
* Corresponding author. Tel.: +86 75526036710.

E-mail address: maoxz@sz.tsinghua.edu.cn (X.-Z. Mao).
}

pollution accidents, and in turn to enable proper emergency response, post-event remediation and liability apportionment. Such problems are generally referred to as river contaminant source identification, and can be formulated as inverse models to estimate missing source release history from the detected contaminant plume at monitoring sites.

Source identification problems are inherently ill-posed and difficult to solve as a result of the irreversibility of contaminant fate and diffusive nature of transport processes, which smooths the contaminant plume gradually and causes the loss in measurements of information concerning the source release. These ill-posed problems are characterized by high sensitivity to the perturbation, number and location of measurement data, and are expected to be solved by stable and efficient inverse models, such as the classic optimization methods.

In the past two decades, many innovative inverse models, including geostatistical and probabilistic modeling and deterministic direct methods, have been proposed for contaminant source identification, especially in groundwater owing to its substantial significance in engineering practices. An extensive review of many sound methods for recovery of the historical contaminant distribution, source location and release history has been presented by Atmadja and Baqtzoglou [3], Michalak and Kitanidis [32] and Bagtzoglou and Atmadja 
[9]. Commonly employed techniques include the Tikhonov regularization method [38], the nonlinear optimization model [2], particlebased methods [1,7], the backward beam equation method [3,4,8] and geostatistical based methods [6,32]. Some other newly emerged techniques have also been under intensive investigation, such as artificial neural networks [37], a global search algorithm with a constrained least squares estimator [40], geostatistical kriging [36] and iterative regularization [23].

As well as extensive studies to enable groundwater pollutant source identification, a few investigations have been also conducted for river pollution which tends to be more advective than in groundwater. Because of strong advection in rivers, pollutants are transported faster and farther due to the dispersion effect, leading to difficulties in detecting the contaminant plume for the recovery of source release information and to computational instability in numerical solutions. Badia et al. [5] established identifiability and stability results for identification of pollution point sources. Boano et al. [12] applied geostatistical method to estimate a spatially distributed source and independent point sources taking into account the influence of dead zones on transport processes. Cheng and Jia [14] established a computational method for identification of the locations and release time of point sources based on the backward location probability density function method. Other studies made attempts to control contaminant releases into rivers and estuaries $[26,33]$ and in a channel flow [25], and to identify contaminant sources in atmospheric advectiondominated transport process $[10,11]$.

The radial basis collocation method ( $\mathrm{RBCM}$ ) has recently emerged as a new inverse model. RBCM employs radial basis functions (RBFs) to approximate multivariate functions based on scattered data [21] and numerical solution of partial differential equations (PDEs) using methods by Kansa [24] and Hon et al. [22]. The RBCM is a domaintype numerical method involving the same single-step solution procedures as in direct problems and can be efficiently applied to the inverse problem. Cheng and Cabral [13] and Mao and Li [31] employed the inverse multiquadric (MQ) as a RBF to solve ill-posed inverse boundary problems for the Laplace equation. As for the timedependent inverse problems, the global space-time RBCM was then constructed for inverse and backward heat conduction problems [28] and for estimations of the spatial plume distribution and source release history in groundwater [29]. Owing to the complexity of the source identification problem, sensitivity analysis associated with the number, location and error level of observations and model parameters was also performed by $\mathrm{Li}$ and Mao $[28,29]$ The advantages of the space-time RBCM mainly lie in its efficiency due to the truly meshfree and non-iterative property, super-convergence and insensitivity to the direction of time. However, no studies have applied the RBCM to solve contaminant source identification problems in advectiondominated rivers to date.

There is still a pending problem in the conventional RBCM: convergence of the numerical solution is sensitive to the free shape parameter determining the shape of the RBFs. As the shape parameter increases (the RBFs tend to be flatter), the error associated with the numerical solutions is reduced exponentially because of more collocation points effectively involved in the interpolation. However, if the shape parameter exceeds a critical value, the coefficient matrix becomes highly ill-conditioned resulting in the error increasing sharply. The optimal value of the shape parameter is strongly problem-dependent $[18,34]$. Several empirical formulations for selection of the shape parameter have been suggested in other investigations $[18,20,21,24,34,35,41]$. For problems of interpolating scattered data, Foley [18] improved the RBF shape parameter based on the root mean square deviation between the MQ and inverse MQ models at a set of test points, and Rippa [34] proposed instead a cross-validation technique for computing the RBF shape parameter by minimizing the cost function based on the residual errors of only the observation data; for solving PDE systems, Roque and Ferreira [35] built the cost function based only on the residual errors of the governing equations, but Tsaia et al. [41] applied the golden search algorithm to choose a good shape parameter based on the residual errors of both the governing equation and Dirichlet boundary conditions. Li and Mao $[28,29]$ investigated convergence of the global space-time MQ solution with respect to the shape parameter and the scaling factor. The results showed that a reasonable (although not necessarily the best) numerical solution exists within a wide range of both parameters, with a V-shaped or U-shaped error profile with respect to the shape parameter and scaling parameter. Accordingly, it is still necessary to search for the best performance of the RBCM in solving the river contaminant source identification problem based on selection of optimal model parameters.

The present study was conducted to extend the global space-time RBCM [29], used for groundwater, to identify space- and time- dependent source releases causing river pollution. To search for the optimal values of the model parameters, the $k$-fold cross-validation technique based on a new cost function was developed. Also, the idea of the global space-time RBF was extended to Hardy's multiquadric (HMQ), inverse multiquadric (IMQ) and Gaussian (GS). This paper is organized as follows. Sections 2 and 3 describe the governing equations for source identification problems in rivers and the numerical procedures employed by the RBCM for the inverse model, respectively. Section 4 introduces the new cost function designed for choosing the model parameters. Section 5 investigates the sensitivity of the cost function to the parameters coupled with the numerical results of two hypothetical cases, and Section 6 shows application to a real polluted river. Finally, conclusions are given in Section 7.

\section{Governing equations}

Let us consider a river region $\Omega$ surrounded by an entire boundary $\delta \Omega$, in which the pollutant concentration $C(x, t)\left(\mathrm{M} \cdot \mathrm{L}^{-3}\right)$ at time $t(\mathrm{~T})$ and position $x(\mathrm{~L})$ is governed by a two-dimensional depthaveraged advection-dispersion equation with a linear reaction term. We assume (or know) that the source release $s(x, t)$ is spatially distributed on the boundary $\Sigma$ and therefore formulated as a part of the boundary conditions, and the initial concentration is specified to be constant. Also, the flow field can be derived from a two-dimensional depth integrated free surface flow model.

The mathematical model for predicting the fate and transport of the contaminant plume can then be described as:

$$
\left\{\begin{array}{l}
\mathbf{L}[C(\mathbf{x}, t)]=\frac{\partial C(\mathbf{x}, t)}{\partial t}-\nabla \cdot[D \nabla C(\mathbf{x}, t)-\mathbf{u} C(\mathbf{x}, t)] \\
\quad+K C(\mathbf{x}, t)=0,(\mathbf{x}, t) \in \mathbf{\Omega} \times\left(t_{0}, t_{\max }\right) \\
C\left(\mathbf{x}, t_{0}\right)=0, \quad \mathbf{x} \in \Omega \\
\mathbf{F}[C(\mathbf{x}, t)]=\mu \frac{\partial C(\mathbf{x}, t)}{\partial n} \\
\quad+\beta C(\mathbf{x}, t)=s(\mathbf{x}, t),(\mathbf{x}, t) \in \Sigma \times\left(t_{0}, t_{\max }\right), \\
\mathbf{G}[C(\mathbf{x}, t)]=\eta \frac{\partial C(\mathbf{x}, t)}{\partial n} \\
\quad+\gamma C(\mathbf{x}, t)=0, \quad(\mathbf{x}, t) \in \partial \Omega \backslash \Sigma \times\left(t_{0}, t_{\max }\right)
\end{array}\right.
$$

where, $\mathrm{L}, \mathrm{F}$ and $\mathrm{G}$ are the differential operators; $\mu, \beta, \eta$ and $\gamma$ are the coefficients determining the type of boundary conditions (i.e., Dirichlet, Neumann or Robin); $D\left(\mathrm{~L}^{2} \bullet \mathrm{T}^{-1}\right), \mathbf{u}\left(\mathrm{L} \bullet \mathrm{T}^{-1}\right)$ and $K\left(\mathrm{~T}^{-1}\right)$ are the dispersion coefficient, flow velocity, and decay factor, respectively; $t_{0}$ and $t_{\max }$ are the initial and end time of the contaminant transport process, respectively.

If the source release $s(x, t)$ is prescribed and the other boundary conditions and initial conditions are properly defined, Eq. (1) leads to a well-posed contaminant transport problem. The concentration $C(x, t)$ can be determined within the domain at any time and location using conventional procedures such as a finite difference method or finite element method. 
When an accidental release of contaminants occurs at site $\Sigma$, the plume may travel downstream and spread out before being monitored. On the one hand, the dispersive property of river flow smooths the contaminant plume gradually and causes some loss of information concerning the source release. On the other hand, strong advection in rivers may lead to the contaminant plume being only partly caught at the observation sites, or not at all. As a result, the information pertaining to the source release history $\mathrm{s}(x, t)$ becomes incomplete or even completely lost. This is a standard river source identification problem that can be addressed using an inverse model to estimate the source release history $\mathrm{s}(x, t)$ as well as the plume distribution $C(x, t)$ from the observation data $Y_{i}^{\mathrm{e}}\left(t_{j}\right)\left(\mathrm{M} \bullet \mathrm{L}^{-3}\right)$ at the sites $x_{i}$ at time $t_{j}$.

The governing equation of the inverse model can be described as:

$$
\left\{\begin{array}{l}
\mathbf{L}[C(\mathbf{x}, t)]=\frac{\partial C(\mathbf{x}, t)}{\partial t}-\nabla \cdot[D \nabla C(\mathbf{x}, t) \\
\quad-u C(\mathbf{x}, t)]+K C(\mathbf{x}, t)=0, \quad(\mathbf{x}, t) \in \Omega \times\left(t_{0}, t_{\max }\right) \\
C\left(\mathbf{x}, t_{0}\right)=0, \quad \mathbf{x} \in \Omega \\
\mathbf{G}[C(\mathbf{x}, t)]=\eta \frac{\partial C(\mathbf{x}, t)}{\partial n}+\gamma C(\mathbf{x}, t)=0,(\mathbf{x}, t) \in \partial \Omega \backslash \Sigma \times\left(t_{0}, t_{\max }\right) \\
C\left(\mathbf{x}_{i}, t_{j}\right)=Y_{i}^{e}\left(t_{j}\right), i=1, \cdots, N_{o b s} ; j=1, \cdots, M_{o b s}
\end{array},\right.
$$

where, $N_{\text {obs }}$ and $M_{\text {obs }}$ are the numbers of observation sites and of observation data at each observation site, respectively.

The identification of the source release history $s(x, t)$, as a part of boundary conditions on specified $\Sigma$, from a limited number of observed concentrations $Y_{i}{ }^{\mathrm{e}}\left(t_{j}\right)$, belongs to the category of inverse boundary value problems from a mathematical viewpoint. It is an ill-posed inverse problem mathematically for two reasons: first, the problem is usually under-determined because only limited observational data are available; second, the error associated with the solution can be significantly magnified by the observation error. Accordingly, it is necessary to develop an efficient and robust algorithm to solve the inverse problem.

\section{Numerical formulation}

\subsection{Global space-time RBF approximation}

Let $r$ be the Euclidean distance $\left\|x-x_{k}\right\|$ from the test point $\mathrm{x}$ to the training point $x_{k}$, and $\varphi(r)$ be a radial function that depends on the distance $\left\|x-x_{k}\right\|$. If a set of $N_{t}$ scattered data $\left\{x_{k}, f\left(x_{k}\right)\right\}$ is given, the unknown function $f(x)$ is approximated by a linear combination of RBFs [20,30].

$f(\mathbf{x})=\sum_{k=1}^{N_{t}} \varphi_{k}(r) \lambda_{k}$

where $\left[\lambda_{k}\right]$ is a set of unknown coefficients to be determined using the given set of data $\left\{x_{k}, f\left(x_{k}\right)\right\}$. Some commonly used RBFs are listed below $[20,30]$

$\left\{\begin{array}{l}\text { Hardy'smultiquadric(HMQ) }: \varphi(r)=\sqrt{1+c^{2} r^{2}} \\ \text { Inversemultiquadric(IMQ) }: \varphi(r)=1 / \sqrt{1+c^{2} r^{2}} \\ \text { Gaussian(GS) }: \varphi(r)=\exp \left(-c^{2} r^{2}\right)\end{array}\right.$.

These globally supported and infinitely differentiable RBFs depend on a single but free shape parameter $c$ controlling the shape of the RBFs. Franke [20] ranked the RBFs as one of the best methods for interpolating continuous functions in terms of accuracy, stability and easy implementation. Madych [30] concluded that these RBFs possess an exponential rate of convergence, and that they could provide a highly accurate numerical solution with a relatively small number of collocation points. Many other studies provide convergence proofs and error estimations when using RBFs in scattered data interpolation and solving PDEs $[19,42]$.
As a domain-type numerical method, the RBCM can be directly applied to inverse problems using the same numerical procedure as for direct problems [13]: a single-step solution of an over-determined system of linear algebraic equations. This truly mesh-free method is therefore much more efficient than iterative optimization schemes together with a finite difference or finite element method, especially for multidimensional problems.

To solve the time-dependent inverse model, a new Euclidian distance, i.e., $\left(\left\|x-x_{k}\right\|^{2}+w^{2}\left\|t-t_{k}\right\|^{2}\right)^{1 / 2}$, where $w$ is a scale parameter, was proposed by normalizing the time dimension to be a pseudo-spatial dimension in $[28,29]$. Taking a two-dimensional problem as an example, the new Euclidean distance is given by:

$r(\mathbf{x}, t)=\sqrt{\frac{\left(x-x_{k}\right)^{2}}{\left(x_{\max }-x_{\min }\right)^{2}}+\frac{\left(y-y_{k}\right)^{2}}{\left(y_{\max }-y_{\min }\right)^{2}}+w^{2} \frac{\left(t-t_{k}\right)^{2}}{\left(t_{\max }-t_{0}\right)^{2}}}$,

where $x_{\min }\left(y_{\min }\right)$ and $x_{\max }\left(y_{\max }\right)$ are the minimum and maximum values in the $x$ - $(y$-) direction coordinates, respectively. The formulations of the global space-time RBFs, i.e., HMQ, IMQ GS, and their spatial and temporal derivatives are presented in Table 1 which will be used in estimating the release histories of a river pollution source in Section 5 for comparison.

With the new Euclidian distance defined by Eq. (5) in the spacetime domain, the behaviors of numerical solutions of the inverse problem are insensitive to the direction of time. The new RBFs change the physical problems somewhat since the time dimension is a naturally one-way coordinate and the space dimension is a two-way coordinate. As a result, the source identification problem can be resolved over the entire space-time domain $\Omega \times\left[t_{0}, t_{\max }\right]$, which leads to the same single-step solution method as for direct problems.

\subsection{Least-square-based RBCM}

In the conventional RBCM, it is necessary to exactly satisfy the governing equations and boundary conditions at the corresponding nodes using the RBF approximation. This leads to a poorly conditioned coefficient matrix and instability of the solution, especially when the observation data contain measurement errors. Mao and $\mathrm{Li}$ [31] introduced the least-square-based RBCM to search for the bestfit solution of the system, to improve the stability of the numerical solution based on double boundary collocation, one for the governing equation and another for the observation and given boundary conditions.

The detailed numerical procedures for the solution to Eq. (2) are as follows.

Step 1 Assuming that $N \times M$ training nodes are of uniform distribution (although an arbitrarily scattered distribution is allowable in principle) in the spatial domain $\Omega$ and time interval $\left[t_{0}, t_{\max }\right]$, the estimated concentration $C_{e}(x, t)$ can be approximated by the global space-time RBFs as:

$C_{e}(\mathbf{x}, t)=\sum_{k=1}^{N \times M} \varphi_{k}(r) \alpha_{k}$

The source $s_{\mathrm{e}}(x, t)$ is then given by:

$S_{e}(\mathbf{x}, t)=\sum_{k=1}^{N \times M} F\left[\varphi_{k}(r)\right] \alpha_{k}$,

where $\left[\alpha_{k}\right]$ are the unknown coefficients to be determined by the collocation method.

Step 2 The concentration $C_{e}(x, t)$ is required to satisfy the governing equation at the set of $N \times M$ test points, which are the same as the set of $N \times M$ training points, in the entire space-time domain $\Omega \times\left[t_{0}\right.$, $\left.t_{\mathrm{max}}\right]$, to satisfy the initial conditions at the set of $N$ test points in the 
Table 1

Global space-time RBFs and their spatial and temporal derivatives.

\begin{tabular}{|c|c|c|c|}
\hline RBFs & HMQ & IMQ & GS \\
\hline $\begin{array}{l}\varphi_{k}(\mathbf{x}, t) \\
\frac{\partial \varphi_{k}(\mathbf{x}, t)}{\partial x} \\
\frac{\partial^{2} \varphi_{k}(\mathbf{x}, t)}{\partial x^{2}} \\
\frac{\partial \varphi_{k}(\mathbf{x}, t)}{\partial t} \\
\end{array}$ & $\begin{array}{l}\sqrt{1+c^{2}\left[\frac{\left(x-x_{k}\right)^{2}}{\left(x_{\max }-x_{\min }\right)^{2}}+\frac{\left(y-y_{k}\right)^{2}}{\left(y_{\max }-y_{\min }\right)^{2}}+w^{2} \frac{\left(t-t_{k}\right)^{2}}{\left(t_{\max }-t_{0}\right)^{2}}\right]} \\
\frac{c^{2}\left(x-x_{k}\right)}{\left(x_{\max }-x_{\min }\right)^{2}} \varphi_{k}^{-1}(\mathbf{x}, t) \\
\frac{c^{2}}{\left(x_{\max }-x_{\min }\right)^{2}} \varphi_{k}^{-1}(\mathbf{x}, t)-\frac{c^{4}\left(x-x_{k}\right)^{2}}{\left(x_{\max }-x_{\min }\right)^{4}} \varphi_{k}^{-3}(\mathbf{x}, t) \\
\frac{c^{2} w^{2}\left(t-t_{k}\right)}{\left(t_{\max }-t_{0}\right)^{2}} \varphi_{k}^{-1}(\mathbf{x}, t)\end{array}$ & $\begin{array}{l}1 / \sqrt{1+c^{2}\left[\frac{\left(x-x_{k}\right)^{2}}{\left(x_{\max }-x_{\min }\right)^{2}}+\frac{\left(y-y_{k}\right)^{2}}{\left(y_{\max }-y_{\min }\right)^{2}}+w^{2} \frac{\left(t-t_{k}\right)^{2}}{\left(t_{\max }-t_{0}\right)^{2}}\right]} \\
-\frac{c^{2}\left(x-x_{k}\right)}{\left(x_{\max }-x_{\min }\right)^{2}} \varphi_{k}^{3}(\mathbf{x}, t) \\
-\frac{c^{2}}{\left(x_{\max }-x_{\min }\right)^{2}} \varphi_{k}^{3}(\mathbf{x}, t)+\frac{3 c^{4}\left(x-x_{k}\right)^{2}}{\left(x_{\max }-x_{\min }\right)^{4}} \varphi_{k}^{5}(\mathbf{x}, t) \\
-\frac{c^{2} W^{2}\left(t-t_{k}\right)}{\left(t_{\max }-t_{0}\right)^{2}} \varphi_{k}^{3}(\mathbf{x}, t)\end{array}$ & $\begin{array}{l}\exp \left\{-c^{2}\left[\frac{\left(x-x_{k}\right)^{2}}{\left(x_{\max }-x_{\min }\right)^{2}}+\frac{\left(y-y_{k}\right)^{2}}{\left(y_{\max }-y_{\min }\right)^{2}}+w^{2} \frac{\left(t-t_{k}\right)^{2}}{\left(t_{\max }-t_{0}\right)^{2}}\right]\right\} \\
-\frac{2 c^{2}\left(x-x_{k}\right)}{\left(x_{\max }-x_{\min }\right)^{2}} \varphi_{k}(\mathbf{x}, t) \\
-\frac{2 c^{2}}{\left(x_{\max }-x_{\min }\right)^{2}} \varphi_{k}(\mathbf{x}, t)+\frac{4 c^{4}\left(x-x_{k}\right)^{2}}{\left(x_{\max }-x_{\min }\right)^{4}} \varphi_{k}(\mathbf{x}, t) \\
-\frac{2 c^{2} W^{2}\left(t-t_{k}\right)}{\left(t_{\max }-t_{0}\right)^{2}} \varphi_{k}(\mathbf{x}, t)\end{array}$ \\
\hline
\end{tabular}

spatial domain $\Omega$, to satisfy the boundary conditions at the set of $N_{\mathrm{b}}$ test points on the boundary $\delta \Omega \backslash \Sigma$ and to satisfy the observation data at the set of $N_{\text {obs }}$ observation sites. Collocating on the corresponding collocation points gives:

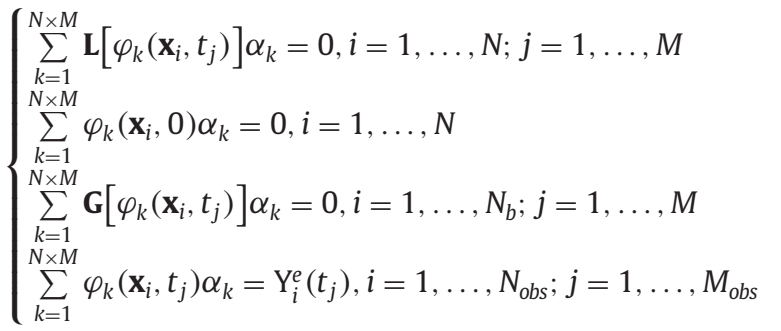

Eq. (8) is an over-determined system of linear algebraic equations with $N \times M$ unknowns and $\left(N \times M+N+N_{\mathrm{b}} \times M+N_{\text {obs }} \times M_{\text {obs }}\right)$ equations.

Step 3 The numerical solution of the system is not required to exactly satisfy all of the governing equations and given conditions. Instead, unknown coefficients $\left[\alpha_{k}\right]$ are required to minimize the residual errors of the linear system. The coefficients $\left[\alpha_{k}\right]$ in Eq. (8) can be determined by minimizing the $L_{2}$ norm using the least-squares method in the following over-determined system:

$\min \left\{\left\|\mathbf{A}\left[\alpha_{k}\right]-\mathbf{b}\right\|^{2}\right\}$,

where $\mathbf{A}$ is the resultant coefficients matrix and $\mathbf{b}$ is the vector with the observation data $Y_{i}{ }^{\mathrm{e}}\left(t_{j}\right)$ at sites $x_{i}\left(i=1,2, \ldots \ldots, N_{\text {obs }}\right)$ at time $t_{j}(j$ $\left.=1,2, \ldots \ldots, M_{\mathrm{obs}}\right)$. The best-fit approximation of the solution $\left[\alpha_{k}\right]$ to system (9) is provided by the singular value decomposition with rank reduction technique [31].

The method relaxes the strong requirement of exact satisfaction of the governing equation and input conditions on the corresponding nodes to improve the conditioning of the coefficient matrix, so that the best solution can be found to approximately match all constraints. The method guarantees the solvability of the ill-posed inverse problem, even with observation error in the input data.

Once the coefficient vectors $\left[\alpha_{k}\right]$ of the linear system are obtained, the plume distribution $C_{\mathrm{e}}(x, t)$ and source release history $\mathrm{s}_{\mathrm{e}}(x, t)$ can be estimated from Eqs. (6) and (7), and the deviations, $E_{C}$ and $E_{S}$, from the true solutions can be given by the root mean square errors:

$$
\begin{aligned}
& E_{C}(c, w)=\sqrt{\frac{1}{N_{C} \times M_{C}} \sum_{i=1}^{N_{C}} \sum_{j=1}^{M_{C}}\left[C_{e}\left(\mathbf{x}_{i}, t_{j}\right)-C_{t}\left(\mathbf{x}_{i}, t_{j}\right)\right]^{2} /} \\
& \sqrt{\frac{1}{N_{C} \times M_{C}} \sum_{i=1}^{N_{C}} \sum_{j=1}^{M_{C}}\left[C_{t}\left(\mathbf{x}_{i}, t_{j}\right)\right]^{2}}, \\
& E_{S}(c, w)=\sqrt{\frac{1}{N_{S} \times M_{S}} \sum_{i=1}^{N_{S}} \sum_{j=1}^{M_{S}}\left[s_{e}\left(\mathbf{x}_{i}, t_{j}\right)-s_{t}\left(\mathbf{x}_{i}, t_{j}\right)\right]^{2} /} \\
& \sqrt{\frac{1}{N_{S} \times M_{S}} \sum_{i=1}^{N_{S}} \sum_{j=1}^{M_{S}}\left[s_{t}\left(\mathbf{x}_{i}, t_{j}\right)\right]^{2}},
\end{aligned}
$$

where, $C_{t}(x, t)$ and $s_{t}(x, t)$ are the true plume distribution and true source release history, respectively, and $N_{C}\left(N_{S}\right)$ and $M_{C}\left(M_{S}\right)$ are the number of the spatial and temporal points for the plume (source), respectively.

\section{Cross-validation technique for model parameters selection}

The global space-time RBCM includes two free model parameters, the shape parameter $c$ and the scaling factor $w$, which need to be prescribed to solve the river contaminant source identification problem. As mentioned in the Introduction, although Li and Mao $[28,29]$ investigated the convergence of the global space-time MQ solution with respect to the shape parameter $c$ and the scaling factor $w$, it is still necessary to seek the best performance of the RBCM based on selection of the optimal model parameters, especially when the method is used in practical applications.

Cross-validation is a statistical technique for model parameter selection that is based on data resampling [15]. In this section, one such common approach, $k$-fold cross-validation based on a new cost function, is proposed to search for near-optimal parameters $c$ and $w$. The new cost function, different from those in $[34,35,41]$, is proposed to combine the residual errors of not only the observed data but also the governing equation, the initial and boundary conditions together at the corresponding points, and then used as the objective function to simulate the deviations of solution (6) and (7) without knowing the true solution. By minimizing the cost function value for the given sets $(c, w)$, we may obtain the near-optimal parameters of the global space-time RBCM.

The unknown coefficients $\left[\alpha_{k}\right]$ are determined in Eq. (9) from the observed data $Y_{i}^{\mathrm{e}}(t)\left(i=1,2, \ldots \ldots, N_{\mathrm{obs}}\right)$, and thus a functional relationship $\Lambda$ can be expressed as follows:

$\left[\alpha_{k}\right]=\Lambda\left\{Y_{1}^{e}\left(t_{j}\right), Y_{2}^{e}\left(t_{j}\right), \ldots, Y_{N_{o b s}}^{e}\left(t_{j}\right) ; j=1, \ldots, M_{o b s}\right\}$

When the $k$-fold cross-validation technique is involved, all of the data $Y_{i}{ }^{\mathrm{e}}\left(t_{j}\right)$ are divided into $k\left\{=N_{\text {obs }}\right\}$ groups, each of which contains the data set for each observation site $x_{i}$. If one data set is removed for validation, say $Y_{m}{ }^{\mathrm{e}}(t)$ at site $x_{m}$, the unknown coefficients $\left[\alpha_{k}{ }^{m}\right]$ can be determined from the remaining $(k-1)$ data sets as follows:

$$
\begin{aligned}
{\left[\alpha_{k}^{m}\right] } & =\Lambda\left\{\mathrm{Y}_{1}^{e}\left(t_{j}\right), \ldots, \mathrm{Y}_{m-1}^{e}\left(t_{j}\right), \mathrm{Y}_{m+1}^{e}\left(t_{j}\right), \ldots, \mathrm{Y}_{N_{o b s}}^{e}\left(t_{j}\right)\right. \\
j & \left.=1, \ldots, M_{o b s}\right\}
\end{aligned}
$$

Subsequently, the plume concentration $C^{m}(x, t)$ can be estimated using the following equation:

$C^{m}(\mathbf{x}, t)=\sum_{k=1}^{N \times M} \varphi_{k}(\mathbf{x}, t) \alpha_{k}^{m}$.

As a result, at the observed site $x_{m}$, the residual error between the observed and computed value is given by $\left|C^{m}\left(x_{m}, t_{j}\right)-Y_{m}{ }^{\mathrm{e}}\left(t_{j}\right)\right|$. Moreover, the residual errors $\left|\mathrm{L}\left[C^{m}\left(x_{i}, t_{j}\right)\right]\right|,\left|C^{m}\left(x_{i}, t_{0}\right)\right|,\left|G\left[C^{m}\left(x_{i}, t_{j}\right)\right]\right|$ of the specified governing equation and initial and boundary conditions at the corresponding points can be obtained. The new cost function in the $L_{1}$ norm is a summation of these $k\left\{=N_{\text {obs }}\right\}$ repeated absolute 

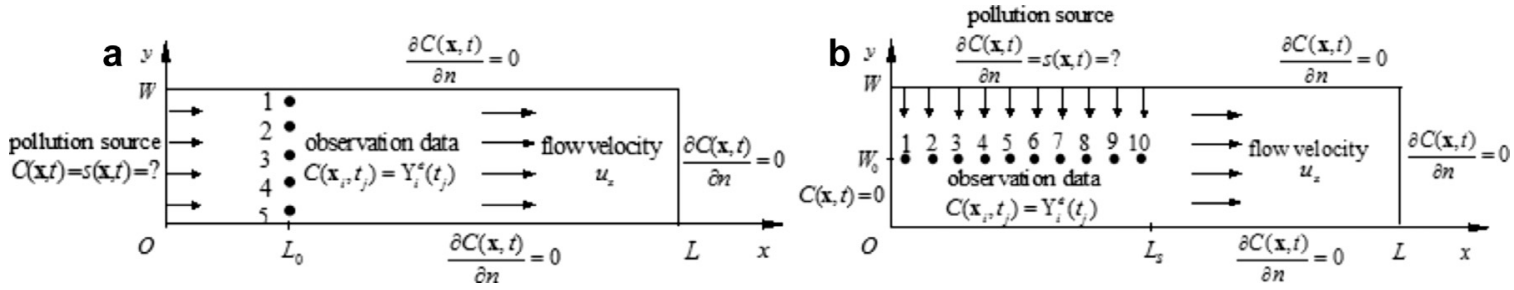

Fig. 1. Unknown pollution source $\mathrm{s}(x, t)$ and observation data $Y_{i}^{\mathrm{e}}\left(t_{j}\right)$ at site $x_{i}$ in Case 1 (a) and Case 2 (b).

residual errors:

$$
\begin{aligned}
\operatorname{COST}(c, w)= & \sum_{m=1}^{k=N_{o b s}}\left\{\sum_{i=1}^{N^{\prime}} \sum_{j=1}^{M^{\prime}}\left|\mathbf{L}\left[C^{m}\left(\mathbf{x}_{i}, t_{j}\right)\right]\right|\right. \\
& +\sum_{i=1}^{N^{\prime}}\left|C^{m}\left(\mathbf{x}_{i}, t_{0}\right)\right|+\sum_{i=1}^{N_{b}{ }^{\prime}} \sum_{j=1}^{M^{\prime}}\left|\mathbf{G}\left[C^{m}\left(\mathbf{x}_{i}, t_{j}\right)\right]\right| \\
& \left.+\sum_{j=1}^{M_{\text {obs }}}\left|C^{m}\left(\mathbf{x}_{m}, t_{j}\right)-\mathrm{Y}_{m}^{e}\left(t_{j}\right)\right|\right\}
\end{aligned}
$$

where $N^{\prime}$ and $M^{\prime}$ are the numbers of test points in the spatial and temporal domain, respectively, and $N_{\mathrm{b}}$ ' is the number of test points on the boundary $\delta \Omega \backslash \Sigma$.

The COST distribution for different sets $(c, w)$ and the near-optimal values of parameters $c$ and $w$ can be obtained by the following procedure.

Step 1 For the given system (9) resulting from the river pollution source identification problem (2), choose the search range $[0$, $\left.c_{\max }\right]$ and $\left[0, w_{\max }\right]$ of parameters $\mathrm{c}$ and $\mathrm{w}$ and the search step $\delta c$ and $\delta w$.

Step 2 For $c=0: \delta c: c_{\max }$ and $w=0: \delta w: w_{\max }$, the cost function values COST for each given set $(c, w)$ are computed, and the $\log _{10}(C O S T)$ profiles are plotted with respect to $c$ and $w$.

Step 3 Select the near-optimal values of parameters $c$ and $w$ that correspond to the minimum COST value on the $\log _{10}(C O S T)$ profile and use the selected values of $c$ and $w$ to solve Eq. (9). Then we can estimate the plume distribution $C_{e}(x, t)$ in (6) and source release history $s_{e}(x, t)$ in $(7)$.

The procedure for near-optimal parameter selection was established according to the new cost function (15). The validity of the method was investigated by numerical experiments and a real pollution incident in the following sections.

\section{Numerical results and discussion}

In this study, two different space- and time- dependent contaminant sources in a two-dimensional river were chosen to investigate the efficiency and accuracy of an inverse model using the global space-time RBCM, especially the method of parameter selection with the new cost function. For simplicity, two hypothetical cases were employed here for methodology testing, but the algorithm can easily be adapted to field conditions.

\subsection{Example problems}

Case 1 is a central source release problem in which the release history of contaminant concentration is unknown. Case 2 is a side source release problem where the release history of contaminant flux is unknown. It is necessary to recover the Dirichlet conditions in Case 1 and the partial Neumann boundary condition in Case 2. Fig. 1 is a schematic plot of the two different release cases. These two different types of space- and time- dependent sources $s(\boldsymbol{x}, t)$ frequently occur in actual contamination accidents. Both cases are considered to estimate river contaminant source release in a confined rectangular river with a steady-state flow field. It is assumed that the transport process of the contaminant plume can be governed by Eq. (2) and is subject to a first-order decay process.

There might be multiple solutions that satisfy the measured data at observation sites; therefore, it is necessary to provide appropriate observation data related to the unknown pollution source. The corresponding observation or monitoring sites $\boldsymbol{x}_{i}$ are shown in Fig. 1. As shown in Fig. 1a, the assumed (or known) position $\Sigma$ of the central source release is at the inlet $x=0$, where the inflow brings a large amount of contaminant into the study area, while the $N_{o b s}$ observation sites are located in the cross section $x=L_{0}$. As shown in Fig. 1b, it is assumed (or known) that a side source is located on the left bank $y=W$ with a length of $L_{s}$. In this scenario, the contaminant plume spreads toward the right bank because of the effect of dispersion, and the $N_{o b s}$ monitoring sites are located at $y=W_{0}$ and cover the same length $L_{s}$. It is assumed that there are $N_{o b s}=5$ and $N_{o b s}=$ 10 observation sites $\mathbf{x}_{i}$ installed at the monitoring section, the spatial coordinates of which are $\left\{x_{i}=L_{0}, y_{i}=20 i-10\right\}$ and $\left\{x_{i}=30 i-15, y_{i}\right.$ $\left.=W_{0}\right\}$ respectively for the two cases, and $M_{\mathrm{obs}}=20$ discrete observation data $Y_{i}^{\mathrm{e}}\left(t_{j}\right)$ at each observation site $x_{i}$. The values of physical parameters are listed in Table 2.

The average Peclet numbers are as high as 300,150 in Case 1 and Case 2, respectively, which means stronger advective effects in rivers than in groundwater systems with Peclet number at 1 [29]. The strong advection in rivers may lead to the contaminant plume being only partly caught at the observation sites, or not at all, and resultant significant ill-posedness of the source identification problem. Moreover, the high Peclet number could cause computational instability or even collapse of the numerical solutions. In both cases, the main flow is in the $x$-direction along the river, so physically the dispersion coefficients in the $x$-direction should be growing with distance faster than in the $y$-direction. Under this circumstance, the pollutant transport in the $y$-direction is mainly due to dispersion. In order to get an enlarging pollution plume, a little larger growing dispersion coefficient in the $y$-direction than in the $x$-direction is used for numerical examples to test the method.

In order to test the stability of the proposed inverse model against observation errors, noisy observation data $\boldsymbol{Y}_{i}{ }^{e}(t)$ at site $\boldsymbol{x}_{i}$ are simulated [2,29,37,39] by:

$\boldsymbol{Y}_{i}^{e}=\boldsymbol{Y}_{i}+e \bullet \boldsymbol{\delta} \bullet \boldsymbol{Y}_{i}, i=1, \ldots, N_{o b s}$,

where $\boldsymbol{Y}_{i}$ is the exact observation data at site $\boldsymbol{x}_{i}$ obtained from the alternating-direction-implicit finite difference model to the direct problem (1) when the true release functions are known; $\delta$ is an array of random numbers subject to standard normal distribution; $e$ is the noise level. A moderate noise level $e=0.05$ is involved in observation data $\boldsymbol{Y}_{i}{ }^{e}(t)$ at site $\boldsymbol{x}_{i}$ (Fig. 2).

In the two cases, $N \times M\{=(21 \times 11) \times 21\}$ training points are uniformly distributed throughout the study river waters $\Omega$ and the time interval $\left[t_{0}, t_{\max }\right]$. All computational codes were written using MATLAB codes, and most of the computational CPU time is used to find the solution to the linear system (9) from the RBCM. The CPU time is about 1800 s on a PC platform with $3.16 \mathrm{GHz}$ CPU and 4.00GB of RAM. 
Table 2

Physical parameter values in test cases (The units of length, time and mass are $L=10 \mathrm{~m}, T=100 \mathrm{~s}, M=100 \mathrm{~g}$ ).

\begin{tabular}{|c|c|c|c|}
\hline \multirow{2}{*}{ Parameter } & \multirow{2}{*}{ Description } & \multicolumn{2}{|l|}{ Value } \\
\hline & & Case 1 & Case 2 \\
\hline$L\left(L_{0}\right)$ & River length (site location) ..................... & $600(150)$ & 600 \\
\hline$W\left(W_{0}\right)$ & River width (site location) ........................ & 100 & $50(25)$ \\
\hline$L_{S}$ & Assumed interval of source distribution........ & - & 300 \\
\hline$\left(t_{\max }-t_{0}\right)$ & Total interval of release history.................. & 200 & 200 \\
\hline$T_{0}$ & Peak time of release history..................... & 40 & 40 \\
\hline$D_{x}$ & Dispersion coefficient in $x$ - direction....... $\mathrm{L}^{2} \bullet \mathrm{T}^{-1}$ & $8+0.002 x$ & $8+0.005 x$ \\
\hline$D_{y}$ & Dispersion coefficient in $y$ - direction...... $\mathrm{L}^{2} \bullet \mathrm{T}^{-1}$ & $6+0.02 y$ & $10+0.05 y$ \\
\hline$u_{x}$ & Flow velocity in $x$ - direction.............. $\bullet \mathrm{T}^{-1}$ & $4+2 \sin (\pi y / 100)$ & $2+\sin (\pi y / 50)$ \\
\hline$K$ & Decay factor..................................... $\mathrm{T}^{-1}$ & 0.002 & 0.002 \\
\hline$s_{t}$ & True source release function.... $\mathrm{M} \bullet \mathrm{L}^{-3}\left(\mathrm{M} \bullet \mathrm{L}^{-4}\right)$ & $10 \exp \left[-20\left(\frac{y-W / 2}{W}\right)^{2}\right] \cdot \frac{t}{T_{0}} \exp \left(-\frac{t^{2}}{2 T_{0}{ }^{2}}\right)$ & $2 \exp \left[-20\left(\frac{x-L_{S} / 2}{L_{S}}\right)^{2}\right] \cdot \frac{t}{T_{0}} \exp \left(-\frac{t^{2}}{2 T_{0}{ }^{2}}\right)$ \\
\hline
\end{tabular}
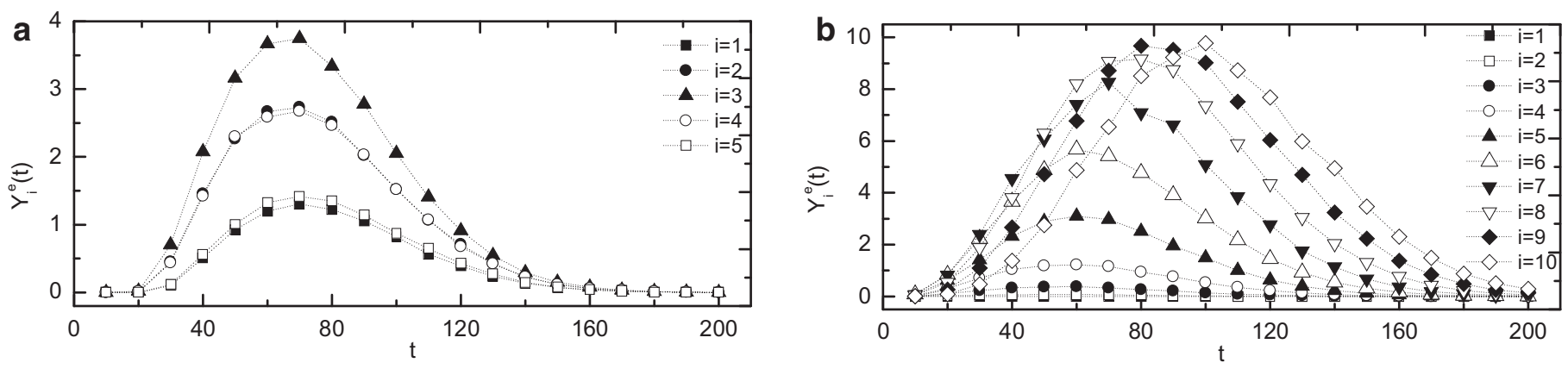

Fig. 2. Observation data $Y_{i}{ }^{\mathrm{e}}(t)$ with noise level $e=0.05$ for Case 1 (a) and Case 2(b).

\subsection{Sensitivity to parameters $c$ and $w$}

To analyze the sensitivity of the global space-time HMQ IMQ and GS solutions to parameters $\mathrm{c}$ and $\mathrm{w}$ in the test cases, we calculated the $E_{C}$ errors on the grid of $N_{C} \times M_{C}\{=(31 \times 21) \times 21\}$ for the estimated concentration data $C_{e}(x, t)$, and the $E_{S}$ errors on the grid of $N_{S} \times M_{S}\{=51 \times 51\}$ for the estimated source data $s_{e}(x, t)$, respectively, and then searched for the minimum values of $E_{C}$ and $E_{S}$ errors corresponding to the optimal values of $c$ and $w$. The distribution of $E_{C}$ and $E_{S}$ errors was calculated for all sets of parameters $(c, w)$ within the ranges of $\left[0, c_{\max }\right]\{=[0,5]\}$ and $\left[0, w_{\max }\right]\{=[0,15]\}$ at intervals of $\delta c\{=0.25\}$ and $\delta w\{=0.5\}$. It should be noted that the prescribed true solution is only used for calculation of the $E_{C}$ and $E_{S}$ errors, but not involved in the COST distribution calculation procedure.

The $\log _{10}\left(E_{C}\right)$ and $\log _{10}\left(E_{S}\right)$ profiles of global HMQ, IMQ and GS solutions with respect to the model parameters $c$ and $w$ for both cases are plotted in Figs. 3 and 4. These figures illustrate that the errors $E_{C}$ and $E_{S}$ vary in a cone-shaped distribution with respect to $c$ and $w$, which is similar to the results reported by Li and Mao [28], and that the global optimal value ranges are as shown in the core of the cone. Interestingly, comparison of the optimal region of $E_{C}$ errors with that of the $E_{S}$ errors (Fig. 3a and b and Fig. $4 \mathrm{a}$ and $\mathrm{b}$ ) revealed that for each RBF solution the $\log _{10}\left(E_{C}\right)$ and $\log _{10}\left(E_{S}\right)$ distribution patterns are very similar. The optimal regions of the $E_{C}$ and $E_{S}$ errors almost overlap with each other, which means that the optimal parameters for the solutions $C_{t}(x, t)$ are almost same as the source release history $s_{t}(x, t)$. These findings support the use of a single cost function as an indicator for both the $E_{C}$ and $E_{S}$ errors. If the optimal region of the cost function as an indicator successfully maps this region, parameter optimization based on both the $E_{C}$ and $E_{S}$ errors can be converted into the problem of optimization based on the cost function alone.

As shown in Fig. 3, for Case 1, the regions bounded by the contour $E_{C}=10^{-1.35}\{=0.0447\}, E_{S}=10^{-0.95}\{=0.1122\}$ are considered the optimal regions for performance of the HMQ IMQ and GS solutions, in which the HMQ, IMQ and GS solutions can attain the same prescribed accuracy. Moreover, the optimal parameter values give $E_{C}\left(E_{S}\right)$ errors of the HMQ, IMQ and GS solutions, 0.0320 (0.0717), 0.0313 (0.0741) and $0.0316(0.0754)$, respectively, at almost the same level of estimation accuracy (Table 3 ). Finally, the optimal parameter ranges of the HMQ and GS solutions are shown to be wider than that of the IMQ solution, which indicates that they are more likely to obtain better estimations of the plume concentration and central source release history.

As shown in Fig. 4, for Case 2, the optimal ranges of the $\log _{10}\left(E_{C}\right)$ and $\log _{10}\left(E_{S}\right)$ subject to the parameters $c$ and $w$ are marked by the contours $E_{C}=10^{-1.1}\{=0.0794\}, 10^{-1.2}\{=0.0631\}$ and $10^{-1.3}\{=0.0501\}$ and $E_{S}=10^{-0.7}\{=0.1995\}, 10^{-0.8}\{=0.1585\}$ and $10^{-0.9}\{=0.1259\}$ for the HMQ IMQ and GS solutions, respectively. The optimal parameter values in Table 3 give $E_{C}\left(E_{S}\right)$ errors, 0.0660 (0.1879), 0.0500 (0.1476) and 0.0349 (0.0939), for the HMQ IMQ and GS solutions, respectively. The optimal parameter range of the GS solution is shown to be larger than that of the IMQ and HMQ solutions. Within the optimal parameter ranges, the GS solution and the estimated source release history are much better than the IMQ and HMQ solutions.

Comparison of both cases using the optimal values of parameters $c$ and $w$ reveals that the $E_{S}$ errors of the side source release, which is a release history of contaminant flux, are larger than the $E_{S}$ errors of the central source release, which is a release history of contaminant concentration. This may be caused by Neumann boundary conditions in which the numerical solutions oscillate more than those on the Dirichlet boundary [27]. Moreover, the $E_{S}$ errors of estimated source release history are slightly larger than the $E_{C}$ errors of estimated plume concentration in both cases. This is because the RBCM is a strong-form mesh-free method with solutions that oscillate more on the boundary than in the domain [17].

\subsection{Practical performance of the $k$-fold cross-validation technique}

Sensitivity analysis can provide insights into the solutions with respect to the parameters, but the practical performance of the RBCM requires determining model parameters without known true solutions. To examine whether the COST distribution varies with 

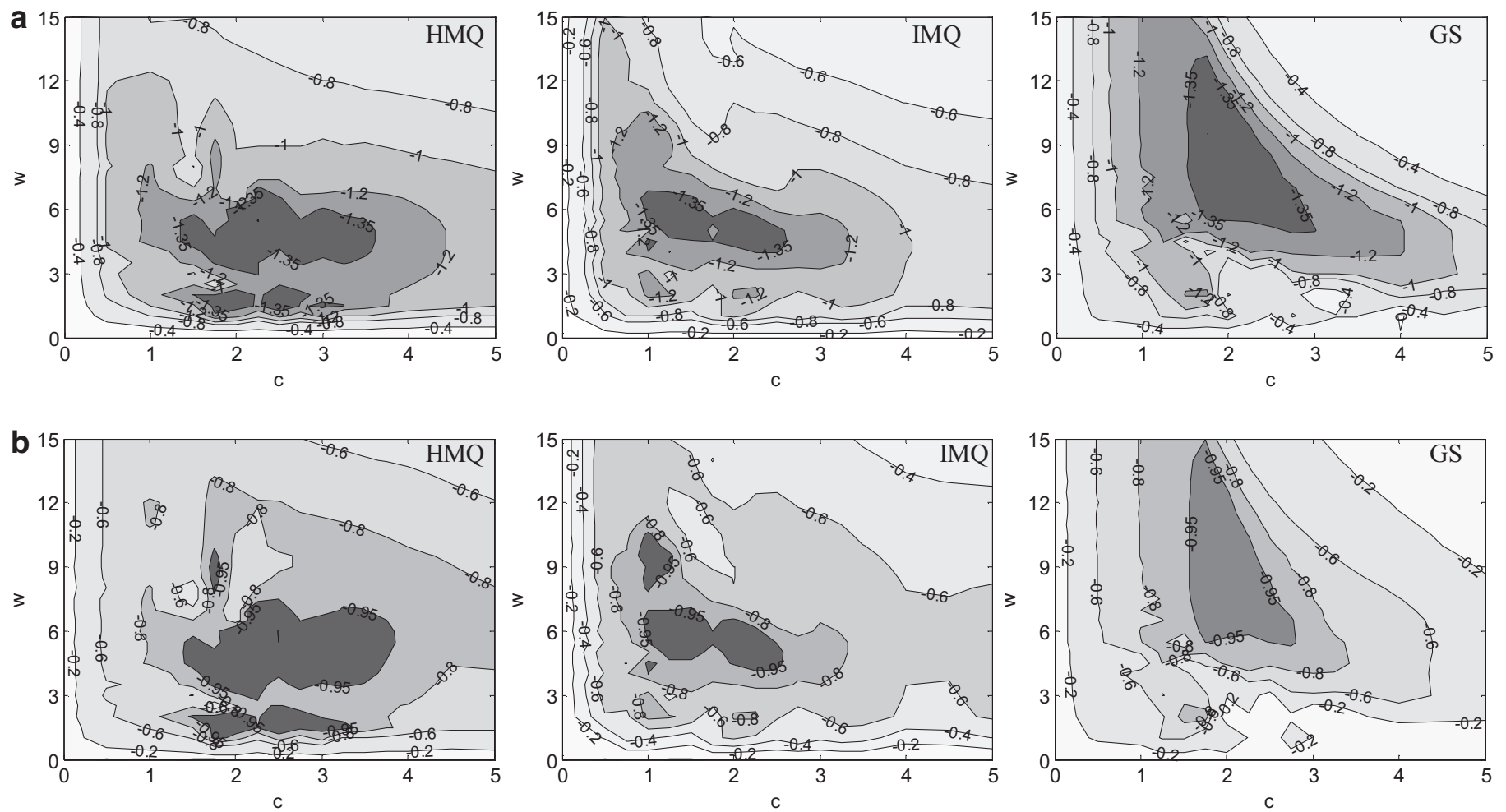

Fig. 3. The $\log _{10}\left(E_{C}\right)(\mathrm{a})$ and $\log _{10}\left(E_{S}\right)(\mathrm{b})$ profiles with respect to $c$ and $w$ in Case 1.

parameters $c$ and $w$ in a similar pattern as the $E_{S}$ and $E_{C}$ profiles, and whether parameters $c$ and $w$ selected by minimizing the COST value are close to the optimal values based on both the $E_{C}$ and $E_{S}$ errors, we computed the COST distribution on the grid $N^{\prime} \times M^{\prime}\{=(20 \times 10) \times 20\}$, subject to the same parameter ranges $\left[0, c_{\max }\right]\{=[0,5]\}$ and $[0$, $\left.w_{\max }\right]\{=[0,15]\}$ and same parameter steps $\delta c\{=0.25\}$ and $\delta w\{=0.5\}$ as were used for sensitivity analysis of the parameters.

The $\log _{10}(C O S T)$ profiles obtained using different global RBFs solutions with respect to parameters $c$ and $w$ are plotted in Fig. 5 for both cases. As shown in the figure, the distribution of $\log _{10}(C O S T)$ for both cases is very regular, varying in a cone-shaped distribution with respect to $c$ and $w$, very similar to the $\log _{10}\left(E_{C}\right)$ and $\log _{10}\left(E_{S}\right)$ distribution in Figs. 3 and 4. These findings indicate that the $\log _{10}(C O S T)$ profiles reasonably repeat the $\log _{10}\left(E_{C}\right)$ and $\log _{10}\left(E_{S}\right)$ profiles, and that the cost function may be used as an indicator to search for reasonable parameter values.

In Case 1 , the region bounded by the contour COST $=10^{1.5}$ in Fig. 5a represents a prescribed low value of the cost function, which is reasonably consistent with the area in which the $E_{C}$ errors are less than $10^{-1.35}$ in Fig. 3. Therefore, this area can be referred to as the near-optimal region. As shown in Table 4, the minimal COST values give near-optimal values of $(c, w)$ as $(2.00,2.0),(1.50,2.0)$ and $(2.75$, 3.0) for HMQ IMQ and GS, respectively. The near-optimal values of $c$ here are very close to the optimal values in Table 3, but those of $w$ are slightly smaller.

The near-optimal parameters give $E_{C}\left(E_{S}\right)$ errors of the HMQ, IMQ and GS solution, 0.0336 (0.0810), 0.0744 (0.1827) and 0.1071 (0.2711), respectively. The numerical results show that the $E_{C}\left(E_{S}\right)$ errors of the HMQ solution obtained using the near-optimal parameters (Table 4) are close to those of the optimal parameters (Table 3), while the accuracies of the GS and IMQ solutions obtained using the near-optimal $c$ and $w$ decrease when the $k$-fold cross-validation technique is used for (near-optimal) parameter selection.

In Case 2, the regions marked by COST $=10^{2.5}, 10^{2.4}$ and $10^{2.3}$ are considered to be the near-optimal regions for the HMQ IMQ and GS solutions, respectively. Comparing Figs. 4 and $5 \mathrm{~b}$, the $\log _{10}(C O S T)$ profiles of the HMQ IMQ and GS solutions with respect to parameters $c$ and $w$ are similar to the $\log _{10}\left(E_{C}\right)$ and $\log _{10}\left(E_{S}\right)$ profiles. The nearoptimal ranges of $c$ and $w$ where the COST values are less than $10^{2.5}$, $10^{2.4}$ and $10^{2.3}$ match the regions in which the $E_{\mathrm{C}}$ errors are less than $10^{-1.1}, 10^{-1.2}$ and $10^{-1.3}$ very well for HMQ, IMQ and GS, respectively. The near-optimal $c$ and $w$ obtained by minimizing the COST values are $(1.25,2.0),(1.25,1.5)$ and $(2.50,1.5)$ for the HMQ IMQ and GS, respectively (Table 4 ), while the optimal $c$ and $w$ obtained from minimizing the $E_{C}$ errors are $(1.25,3.0),(1.25,3.0)$ and $(3.00,1.0)$ for the HMQ IMQ and GS, respectively (Table 3 ). The "near-optimal" values of $c$ and $w$ tend to be the optimal values.

The $E_{C}\left(E_{S}\right)$ errors of the HMQ IMQ and GS solutions obtained using the near-optimal $c$ and $w$ were 0.0677 (0.1873), $0.0526(0.1411)$ and $0.0430(0.1245)$, respectively, almost the same as the $E_{C}\left(E_{S}\right)$ errors obtained using the optimal values of $c$ and $w$. The HMQ IMQ and GS solutions obtained using the $k$-fold cross-validation technique enable the reasonable performances in practice. The GS solution produced the best estimates for realistic situations in which the true solutions are unknown.

\subsection{Estimation of the contaminant release and plume distribution}

The estimated contaminant release histories and plume distributions using the near-optimal parameters with the $k$-fold crossvalidation technique for both test cases were compared with the true solutions to further validate the applicability of the RBCM. For the purpose of brevity, we only present the estimation of the HMQ solution (Fig. 6) in Case 1 and of the GS solution (Fig. 7) in Case 2 here. The comparison focuses on the peak source strength $s_{\text {peak }}$ occurring at time $T_{0}$ and the contaminant plume at $t *=50$ and 100 . The $E_{S}$ and $E_{C}$ errors and the peak values at $t *=50$ and 100 for HMQ, IMQ and GS solutions for both cases are listed in Tables 5 and 6.

For Case 1, the central source, the contaminant release function has its peak value $s_{\text {peak }}=6.0653$ at $\left\{x=(0, \mathrm{~W} / 2), t=T_{0}\right\}$. The peak 

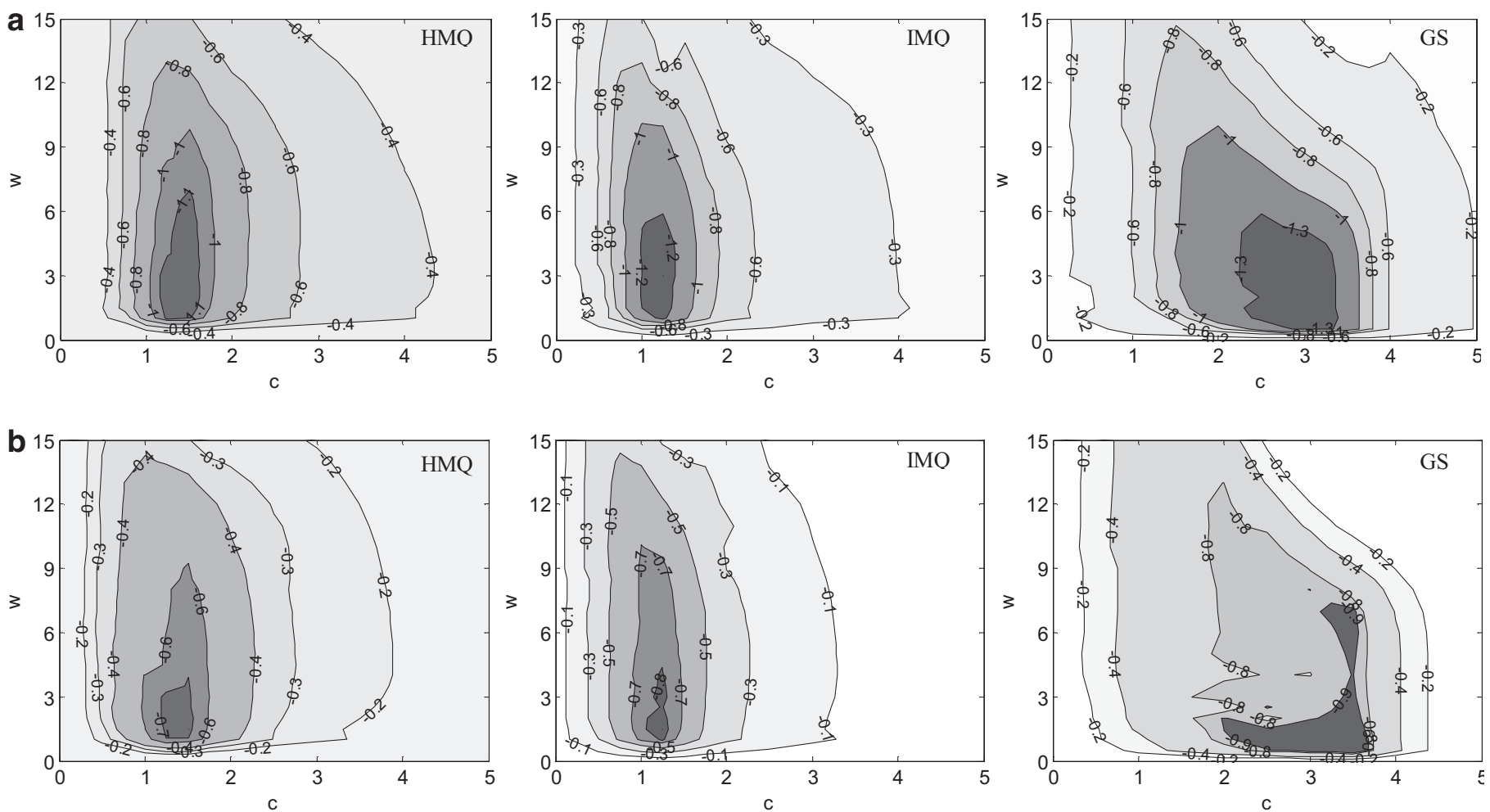

Fig. 4. The $\log _{10}\left(E_{C}\right)(\mathrm{a})$ and $\log _{10}\left(E_{S}\right)(\mathrm{b})$ profiles with respect to $c$ and $w$ in Case 2 .

Table 3

The optimal value of $\mathrm{c}$ and $\mathrm{w}$ obtained from minimizing the $E_{\mathrm{C}}$ error.

\begin{tabular}{|c|c|c|c|c|c|c|}
\hline & \multicolumn{3}{|l|}{ Case 1} & \multicolumn{3}{|l|}{ Case 2} \\
\hline & $(c, w)_{\text {optimal }}$ & $E_{C}$ & $E_{S}$ & $(c, w)_{\text {optimal }}$ & $E_{C}$ & $E_{S}$ \\
\hline HMQ & $(2.00,5.0)$ & 0.0320 & 0.0717 & $(1.25,3.0)$ & 0.0660 & 0.1879 \\
\hline IMQ & $(1.25,5.5)$ & 0.0313 & 0.0741 & $(1.25,3.0)$ & 0.0500 & 0.1476 \\
\hline GS & $(1.75,9.5)$ & 0.0316 & 0.0754 & $(3.00,1.0)$ & 0.0349 & 0.0939 \\
\hline
\end{tabular}

Table 4

The near optimal value of $\mathrm{c}$ and $\mathrm{w}$ obtained from minimizing the COST value.

\begin{tabular}{|c|c|c|c|c|c|c|}
\hline & \multicolumn{3}{|l|}{ Case 1} & \multicolumn{3}{|l|}{ Case 2} \\
\hline & $(c, w)_{\text {chosen }}$ & $E_{C}$ & $E_{S}$ & $(c, w)_{\text {chosen }}$ & $E_{C}$ & $E_{S}$ \\
\hline HMQ & $(2.00,2.0)$ & 0.0336 & 0.0810 & $(1.25,2.0)$ & 0.0677 & 0.1873 \\
\hline IMQ & $(1.50,2.0)$ & 0.0744 & 0.1827 & $(1.25,1.5)$ & 0.0526 & 0.1411 \\
\hline GS & $(2.75,3.0)$ & 0.1071 & 0.2711 & $(2.50,1.5)$ & 0.0430 & 0.1245 \\
\hline
\end{tabular}

release time is 40 , and the peak concentrations of the plume at time $t *=50$ and 100 are $C_{\text {peak }}=5.7229$ and 2.7326 at $x=(0,50)$ and $(305$, 50 ), respectively. As shown in Fig. 6, the peak entered the computational domain at $t *=50$, and most of the contaminant was released into the domain by $t *=100$. Although the $E_{S}$ errors of the HMQ, IMQ and GS solutions are $0.0810,0.1827$ and 0.2711 , the estimated peak source values are $s_{\text {peak }}=6.1111,6.1831$ and 6.3469, respectively, and the relative errors of the peak values are less than $5 \%$. The estimated release histories recovered the spatial distributions of the pollution source and peak time $T_{0}$ very accurately, and the HMQ IMQ and GS solutions provide a reasonable estimation of the release history.

The RBCM provides an accurate estimation of the plume distributions at time $t *=50$ and 100 . The $\left.E_{C}\right|_{t_{*}=50}\left(\left.E_{C}\right|_{t_{*}=100}\right)$ errors of the HMQ, IMQ and GS solutions are $0.0212(0.0165), 0.0296$ (0.0146) and 0.0383 (0.0145), respectively. The estimated peak concentrations of the plume of the HMQ IMQ and GS solutions at time $t *=50(100)$ are
$C_{\text {peak }}=5.9833$ (2.7553), 6.0709 (2.7606) and 6.1253 (2.7585), which had relative derivations from the true values of $4.55 \%(0.83 \%), 6.08 \%$ (1.02\%) and $7.03 \%(0.95 \%)$, respectively. All of the estimated plume distributions identify the location of the peak plume concentration very accurately.

Similar results were shown for Case 2, the side source. The release flux function reaches a peak value $s_{\text {peak }}=1.2131$ at $\left\{x=\left(L_{S} / 2, W\right), t\right.$ $\left.=T_{0}\right\}$, and the peak concentrations of the plume at time $t *=50$ and 100 are $C_{\text {peak }}=23.5788$ and 12.2846 at $x=(175,50)$ and $(250,50)$, respectively. As shown in Fig. 7 , the peak entered the computational domain at $t *=50$, and most of the contaminant was released into the domain by $t *=100$. The estimated release history values $\mathrm{s}_{\mathrm{e}}(x, t)$ are in reasonable agreement with the actual ones, and recover the spatial distributions of pollution source and peak time $T_{0}$ very accurately. The $E_{S}$ errors of the HMQ, IMQ and GS solutions are $0.1873,0.1411$ and 0.1245 , respectively, but the estimated peak flux of the HMQ IMQ and GS solutions are $s_{\text {peak }}=1.0640,1.1169$ and 1.2541 , respectively, which have individual relative errors of $12.29 \%, 7.93 \%$ and $3.38 \%$. Estimation of the release history using the GS outperforms those obtained using IMQ and HMQ, and the GS solution offers higher accuracy of the estimation of the plume distributions at time $t *=50$ and 100 than the IMQ and HMQ solutions.

Moreover, all estimated plume distributions identify the location of the plume peak concentration very accurately. The $\left.E_{C}\right|_{t *=50}$ $\left(\left.E_{C}\right|_{t_{*}=100}\right)$ errors of the HMQ, IMQ and GS solutions are 0.1073 $(0.0350), 0.0813(0.0258)$ and $0.0463(0.0306)$, respectively, and the estimated peak plume concentrations of the HMQ IMQ and GS solutions at time $t *=50$ (100) are $C_{\text {peak }}=21.5026$ (12.7245), 22.0698 (12.6684) and 24.1468 (12.6165), respectively, with individual relative errors of $8.81 \%(6.40 \%), 6.08 \%$ (1.02\%) and $7.03 \%$ (2.41\%). The estimated plume distributions $C_{e}(x, t *)$ at time $t *=50$ and 100 using the global space-time HMQ, IMQ and GS are in good agreement with the true values.

The numerical experiments of both cases show that, with parameter selection using the new cost function (15), the accuracy of 

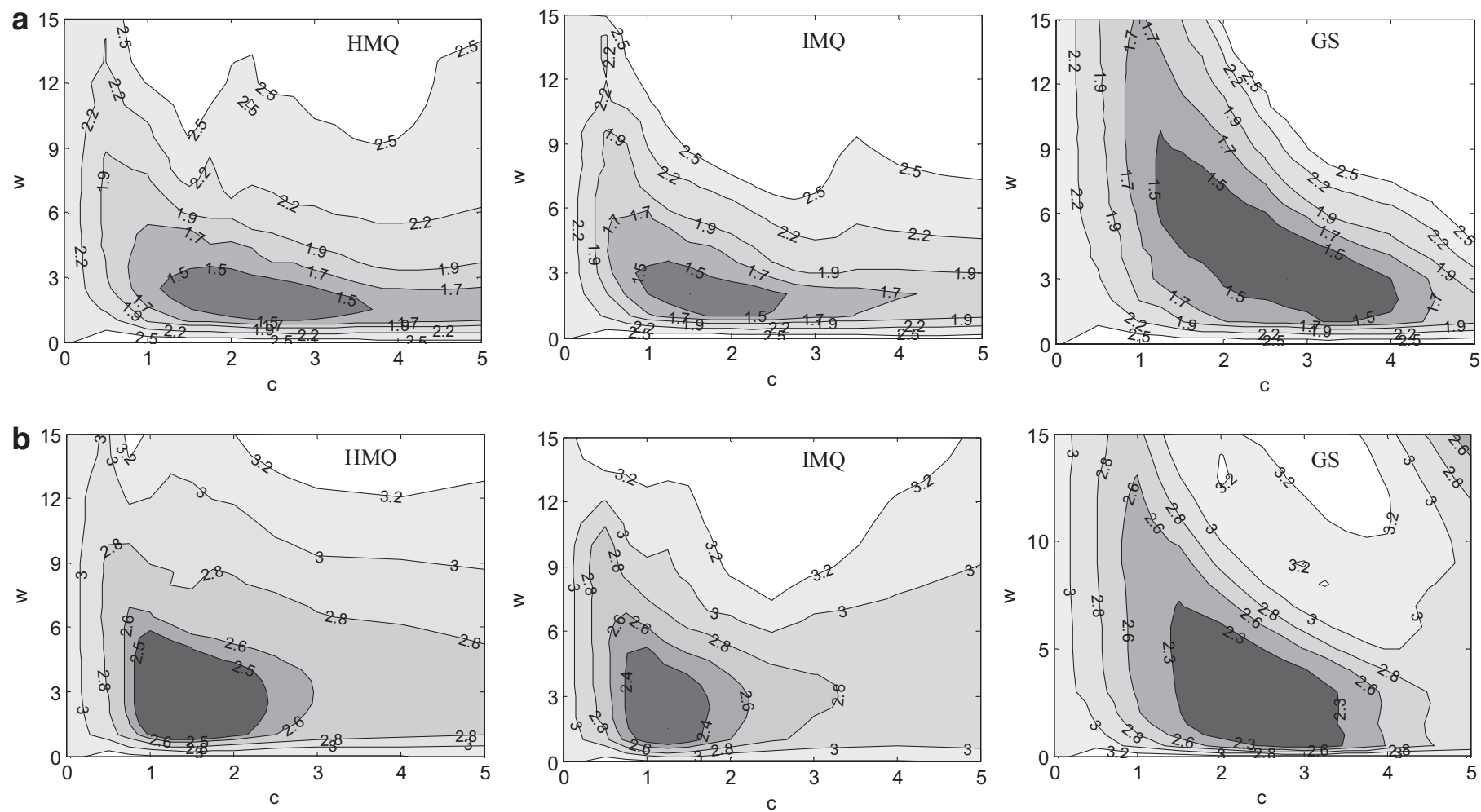

Fig. 5. The $\log _{10}(C O S T)$ profiles with respect to $c$ and $w$ for Case $1(\mathrm{a})$ and 2 (b).
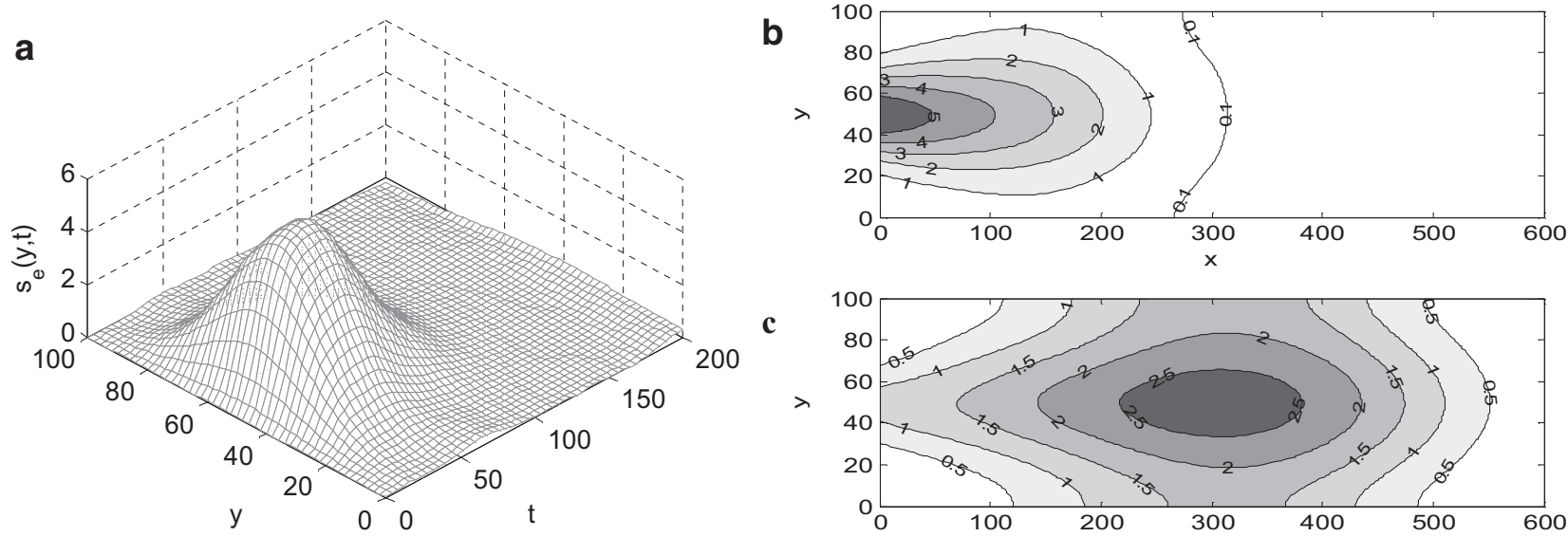

c

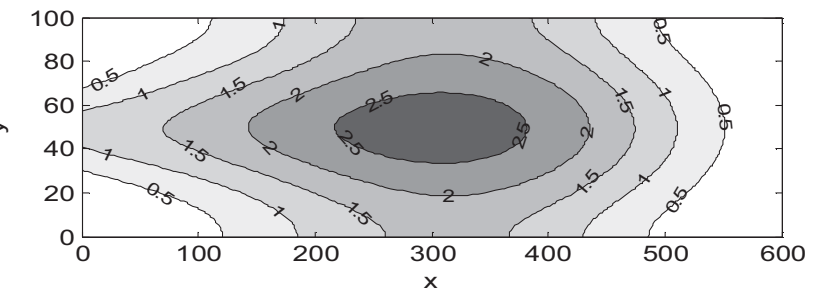

Fig. 6. $s_{e}(x, t)(\mathrm{a})$ and $C_{e}\left(x, t^{*}\right)$ at $t^{*}=50(\mathrm{~b})$ and $t^{*}=100$ (c) estimated using HMQ for Case 1 .

numerical solutions for the plume distribution and the contaminant release history are the same as those for the input observation data.

\section{Application to a real polluted river}

In order to investigate the performance of the model in practical applications, a real river pollution accident was used as a case study to estimate the contaminant release history with the global spacetime RBCM and $k$-fold cross-validation technique.

A nitrobenzene-pollution accident (Fig. 8a) happened in a river in Northeastern China in 2005 [43]. The length of the study area in the river is roughly $L=120 \mathrm{~km}$ from upstream site A to downstream site $\mathrm{B}$, and the observation of nitrobenzene concentrations (Fig. 8b) was carried out after the accident over a span of $T=210 \mathrm{~h}$ between 21:00 November 23rd and 15:00 December 2nd. The observed data were obtained from [43]. In this test of computation by the RBCM model, the observed data $s_{t}(t)$ at site $A$ is supposed to be the unknown release history and may be estimated using the observed data $\mathrm{Y}^{e}(t)$ at site $\mathrm{B}$, and the biodegradation process of nitrobenzene is not taken into accounts. The average width, depth and flow velocity of the reach were about $300 \mathrm{~m}, 1.5 \mathrm{~m}$ and $1.25 \mathrm{~km} / \mathrm{h}$, respectively. The dispersion coefficient was $1.15 \mathrm{~km}^{2} / \mathrm{h}$ determined by the calibrations. The Peclet number of the problem is approximately 130 , the same order of magnitude as in Section 5 numerical cases.

The 1-D HMQ-structured RBCM model was employed on the uniform grid of $N \times M\{=21 \times 31\}$ training points. Using the new cost function (15), the COST distribution subject to the parameters $c$ and $w$ in the ranges of $\left[0, c_{\max }\right] \times\left[0, w_{\max }\right]\{=[0,10] \times[0,5]\}$ is presented in Fig. 9a. It is easy to find that the region with lower COST values is marked by the contour COST $=10^{0.32}$, which is supposed to enclose the near-optimal ranges of $c$ and $w$. The near-optimal parameters are chosen as $(c, w)=(5.25,1.5)$ that corresponds to the minimum COST 

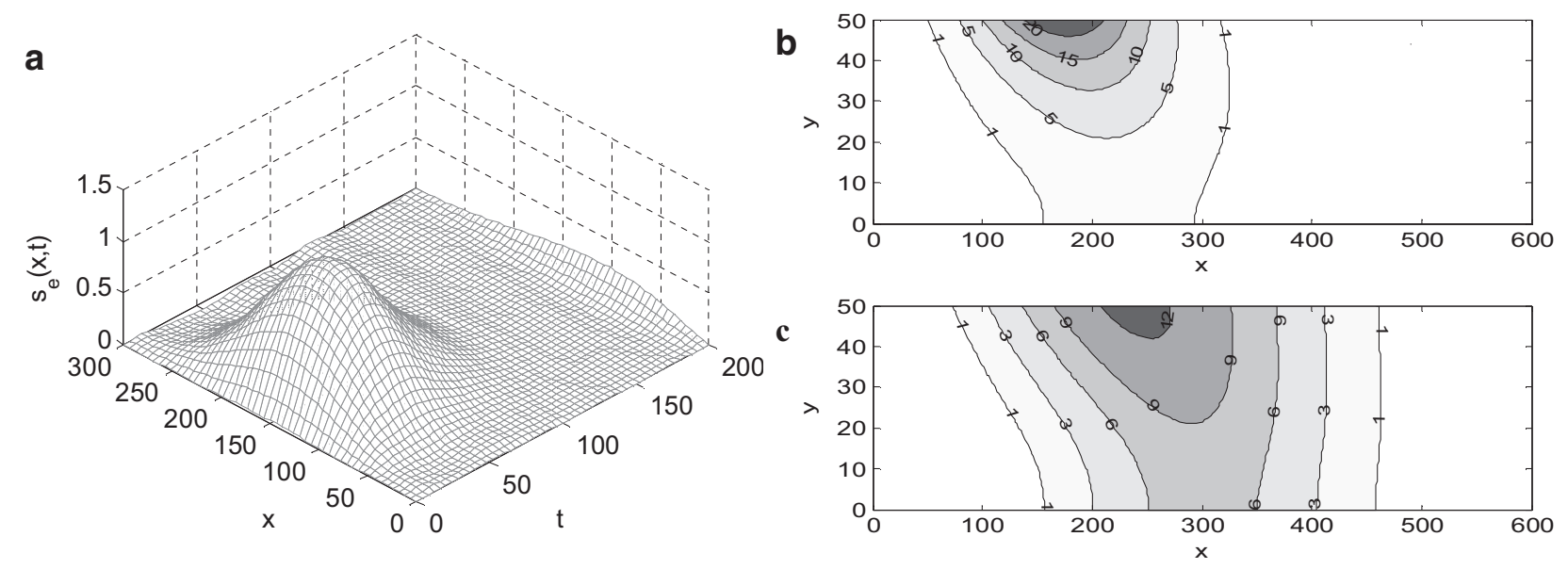

Fig. 7. $s_{e}(x, t)(\mathrm{a})$ and $C_{e}\left(x, t_{*}\right)$ at $t *=50(\mathrm{~b})$ and $t *=100(\mathrm{c})$ estimated using GS for Case 2 .

Table 5

The estimated results using the chosen model parameters $c$ and $w$.

\begin{tabular}{|c|c|c|c|c|c|c|c|c|}
\hline & \multicolumn{4}{|l|}{ Case 1} & \multicolumn{4}{|l|}{ Case 2} \\
\hline & $(c, w)_{\text {chosen }}$ & $E_{S}$ & $\left.E_{C}\right|_{t *=50}$ & $\left.E_{C}\right|_{t *=100}$ & $(c, w)_{\text {chosen }}$ & $E_{S}$ & $\left.E_{C}\right|_{t *=50}$ & $\left.E_{C}\right|_{t *=100}$ \\
\hline HMQ & $(2.00,2.0)$ & 0.0810 & 0.0212 & 0.0165 & $(1.25,2.0)$ & 0.1873 & 0.1073 & 0.0350 \\
\hline IMQ & $(1.50,2.0)$ & 0.1827 & 0.0296 & 0.0146 & $(1.25,1.5)$ & 0.1411 & 0.0813 & 0.0258 \\
\hline GS & $(2.75,3.0)$ & 0.2711 & 0.0383 & 0.0145 & $(2.50,1.5)$ & 0.1245 & 0.0463 & 0.0306 \\
\hline
\end{tabular}

Table 6

The estimated peak results using the chosen model parameters $c$ and $w$.

\begin{tabular}{|c|c|c|c|c|c|c|c|c|}
\hline & \multicolumn{4}{|l|}{ Case 1} & \multicolumn{4}{|l|}{ Case 2} \\
\hline & true & HMQ & IMQ & GS & true & HMQ & IMQ & GS \\
\hline$T_{0}$ & 6.0653 & 6.1111 & 6.1831 & 6.3469 & 1.2131 & 1.0640 & 1.1169 & 1.2541 \\
\hline$t *=50$ & 5.7229 & 5.9833 & 6.0709 & 6.1253 & 23.5788 & 21.5026 & 22.0698 & 24.1468 \\
\hline$t *=100$ & 2.7326 & 2.7553 & 2.7606 & 2.7585 & 12.2846 & 12.7245 & 12.6684 & 12.6165 \\
\hline
\end{tabular}
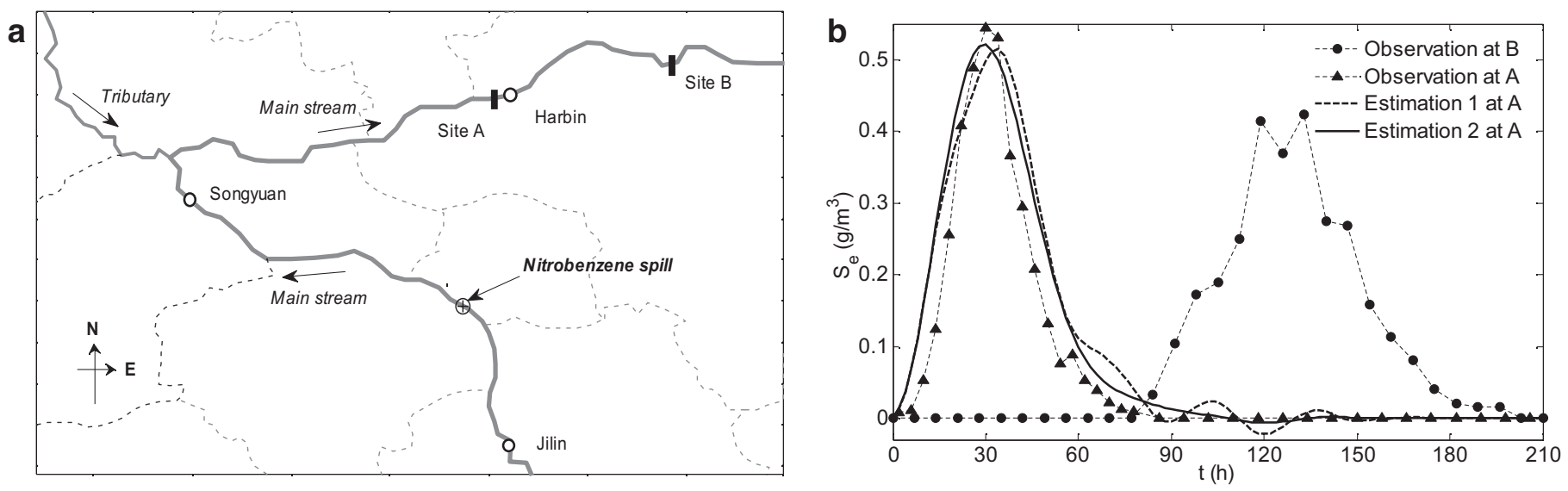

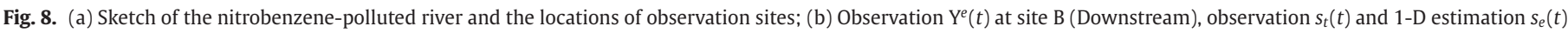
at site A (Upstream).

value. As shown in Fig. 8b, with the chosen model parameters, the estimated release history $s_{e}(t)$ (Estimation 1 in Fig. 8 b) reasonably recovers the nitrobenzene concentration at upstream site A from $t=$ $0 \mathrm{~h}$ to $t=80 \mathrm{~h}$, the peak concentration $C_{\max }=0.5144 \mathrm{~g} / \mathrm{m}^{3}$ with relative error of $5.46 \%$ and the peak time $t=33 \mathrm{~h}$ with a 3-hour delay (observed as peak concentration $C_{\max }=0.5441 \mathrm{~g} / \mathrm{m}^{3}$ at time $t=30 \mathrm{~h}$ shown by $\left.s_{t}(t)\right)$. The new cost function (15), constructed for the $k$-fold cross-validation, aims to find the global optimal numerical results. There may be small ripples when the result is constant (during $t=$
90-150 h in Fig. 8b). The optimal parameters depend on how much measurement data are available, and the error may be eliminated by using sufficient observation data [28]. Also if more training points are used, the ripple effect is expected to decrease. As we see in Fig. 8b, the ripple effect in Estimation 2 is greatly improved when the number of spatial training points $N=41$.

Also two-dimensional hydrodynamic and pollutant transport models were applied to simulate the hydrodynamic conditions and the contaminant source release processes between the sections A 

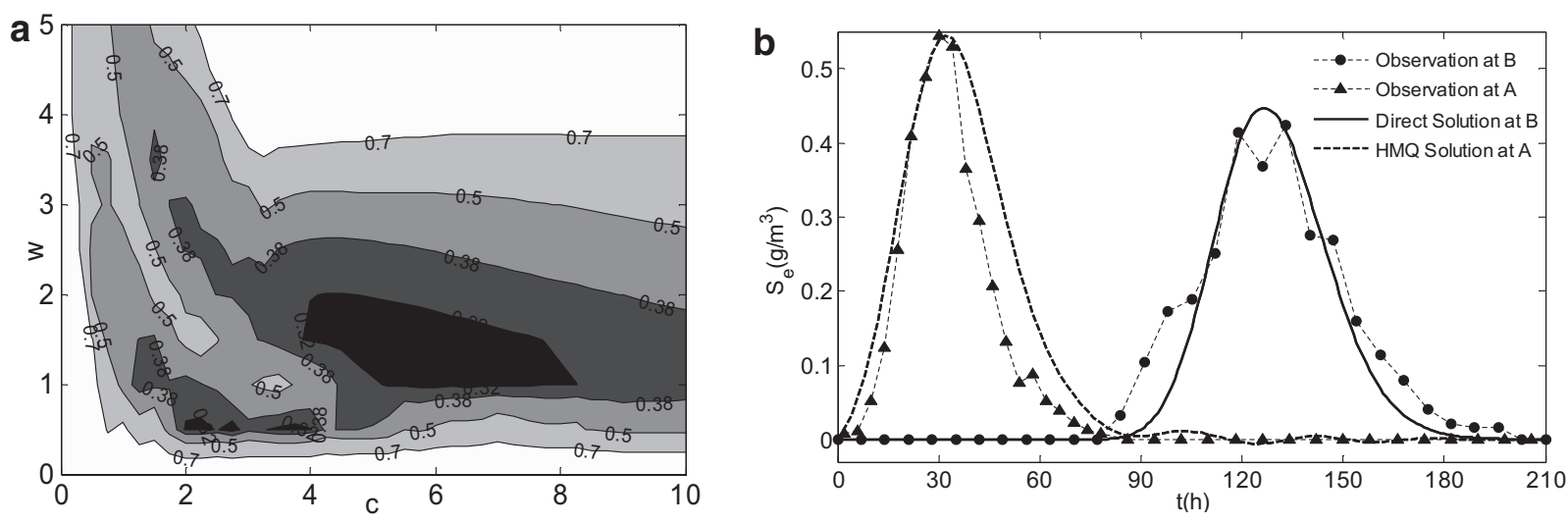

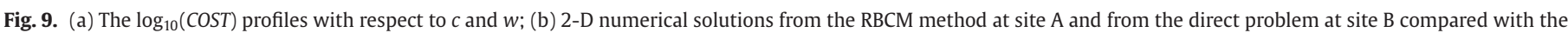
observations.

and B using the Delft3d model, which was developed by Deltares, the Netherlands. The computational grid is $496 \times 11$. For the hydrodynamic model, the flow rate and water level were given as boundary conditions at the upstream and downstream cross-sections A and B respectively. A $k-\varepsilon$ turbulence model was used in the hydrodynamic model. Based on the hydrodynamic results, the nitrobenzene concentration was simulated according to two different cases: direct and inverse. In the direct problem, the observed data $s_{t}(t)$ at site A was supposed to be known as the contaminant release history to calculate the concentration at site B based on the hydrodynamic results using the Delft3D model. In the inverse problem, the RBCM model was used to estimate the unknown release history $s_{t}(t)$ at site A using the observed data $Y_{e}(t)$ at site B with the selected optimal parameters in Fig. 9a. The spatial grid of training points is $100 \times 5$. The hydrodynamic conditions and dispersion coefficients at the training points were extracted from the Delft3d results. The numerical results for both cases are shown in Fig. 9 b. The numerical results from the direct problem match well with the observed data at site B if the source release history is known. The estimated release history $s_{e}(t)$ from the RBCM model recovers the nitrobenzene concentration history very well at site A from $t=0 \mathrm{~h}$ to $t=30 \mathrm{~h}$, and over-estimates it from $t=30 \mathrm{~h}$ to $t$ $=80 \mathrm{~h}$ compared with the observed data. The RBCM model estimates the peak time with a 2-hour delay and the peak concentration for the contaminant history very well.

The application to field concentration data demonstrates that, with selected parameters using the new cost function (15), the RBCM method can reasonably estimate the peak time and concentration for contaminant release history.

\section{Conclusions}

In this study, an inverse model based on the global space-time RBCM was developed for identification of space- and time- dependent pollution sources in advection-dominated rivers. The cost function constructed by the $k$-fold cross-validation technique was proposed to search for model parameters without a known true solution. Two different space- and time- dependent contaminant sources in a two-dimensional river were adopted for methodology testing: a central source problem with a release history of contaminant concentration (the Dirichlet condition) and a side source problem with a release history of contaminant flux (partial Neumann condition). Three global space-time RBFs, HMQ, IMQ and GS, were also compared in two hypothetical cases. The major conclusions are summarized below.

A new cost function was constructed to search the optimal shape parameter and scaling factor of the model. The developed function employs the $k$-fold cross-validation technique based on the residual errors of not only the observed data but also the specified governing equation, the initial and boundary conditions. When the true solutions are unknown, the cost function is a good indicator for seeking the near-optimal parameters using the global space-time RBCM method. The distribution of $\log _{10}(C O S T)$ for both cases varies in a cone-shaped distribution, similar to the $\log _{10}\left(E_{C}\right)$ and $\log _{10}\left(E_{S}\right)$ distribution with respect to $c$ and $w$. The near-optimal parameters can be optimized by minimizing the value of the cost function.

The numerical results for the release history of concentration are better than those for the release history of flux when the optimal parameters are used. This is because the Neumann boundary may have a stronger oscillation than the Dirichlet condition because of the strong-form collocation method. Furthermore, for the central source release, the HMQ solution produces more accurate estimated results than the GS and IMQ solution; meanwhile, for the side source release, the GS solution offers better estimations than the IMQ and HMQ solutions.

The model can identify the source contaminant release histories as well as the plume distribution from the observation data. Two numerical cases demonstrated that the accuracies of numerical solutions for the plume distribution and the contaminant release history are similar to the accuracy of the input data, but the estimated plume distribution is more accurate than the estimated release history; the application to a real polluted river demonstrated that the RBCM method with the new cost function can reasonably estimate the peak time and concentration for contaminant release history, as well as the peak location and concentration for the plume's spatial distribution.

The global space-time RBCM method reduces the pollution source identification problem to a single-step solution of an overdetermined linear system using the same inversion procedures as in the direct problem. The numerical cases and applications to field data validate that the method together with $k$-fold cross-validation is a robust, stable and efficient technique for space- and time- dependent pollution source identification problems in advective river flows. An efficient search algorithm for finding the minimum COST value that corresponds to the near-optimal parameters should be further established to enhance the capability of the RBCM model.

\section{Acknowledgments}

This study was supported by the National Natural Science Foundation of China (no. 41176001), Shenzhen Science and Technology Plan project of China (JCYJ20140902110354253), and Shenzhen Key Laboratory for Coastal Ocean Dynamic and Environment (ZDSY20130402163735964), Graduate School at Shenzhen, Tsinghua 
University, China. We thank the editors and anonymous reviewers for their thorough reviews and constructive remarks.

\section{References}

[1] Ababou R, Bagtzoglou AC, Mallet A. Anti-diffusion and source identification with the RAW scheme: a particle-based censored random walk, J. Environ Fluid Mech 2010;10(1-2):41-76. http://dx.doi.org/10.1007/s10652-009-9153-4.

[2] Aral MM, Guan J, Maslia ML. Identification of contaminant source location and release history in aquifers, J. Hydrol Eng 2001;6(3):225-34. http://dx.doi.org/10. 1061/(ASCE)1084-0699(2001)6:3(225).

[3] Atmadja J, Baqtzoglou AC. State of the art report on mathematical methods for groundwater pollution source identification. Environ Forens 2001;2(3):205-14. http://dx.doi.org/10.1006/enfo.2001.0055.

[4] Atmadja J, Bagtzoglou AC. Pollution source identification in heterogeneous porous media. Water Resour Res 2001;37(8):2113-25. http://dx.doi.org/10.1029/ 2001WR000223.

[5] Badia AE, Ha-Duong T, Hamdi A. Identification of a point source in a linear advection-dispersion-reaction equation: application to a pollution source problem. Inverse Probl 2005;21(3):1121-36. http://dx.doi.org/10.1088/0266-5611/21/ $3 / 020$.

[6] Bagtzoglou AC, Dougherty DE, Tompson AFB. Application of particle methods to reliable identification of groundwater pollution sources. Water Resour Manage 1992;6(1):15-23. http://dx.doi.org/10.1007/BF00872184.

[7] Bagtzoglou AC. On the nonlocality of reversible-time particle tracking methods. Environ Forens 2003;4(3):215-25. http://dx.doi.org/10.1080/713848511.

[8] Bagtzoglou AC, Atmadja J. Marching-jury backward beam equation and quasireversibility methods for hydrologic inversion: application to contaminant plume spatial distribution recovery. Water Resour Res 2003;39(2) 1038: 10-1-10-14 http://dx.doi.org/10.1029/2001WR001021.

[9] Bagtzoglou AC, Atmadja J. Mathematical methods for hydrologic inversion: the case of pollution source identification, chapter in the handbook of environmenta chemistry, water pollution series. Heidelberg-New York: Springer-Verlag; 2005. 5 (F) p. 65-96.

[10] Bagtzoglou AC, Baun SA. Near real-time atmospheric contamination source identification by an optimization-based inverse method. Inverse Probl Sci Eng 2005;13(3):241-59. http://dx.doi.org/10.1080/10682760412331330163.

[11] Bagtzoglou AC, Kenney ED, Hiscox A, Miller DR. Optimization-based atmospheric plume source identification. Environ Forens 2014;15(2):147-58. http://dx.doi. org/10.1080/15275922.2014.890146.

[12] Boano F, Revelli R, Ridolfi L Source identification in river pollution problems: a geostatistical approach. Water Resour Res 2005;41(7) W07023:1-13, http://dx. doi.org/1029/2004WR003754.

[13] Cheng AH-D, Cabral JJSP. Direct solution of ill-posed boundary value problems by radial basis function collocation method. Int J Numer Meth Eng 2005;64(1):4564. http://dx.doi.org/10.1002/nme.1362.

[14] Cheng WP, Jia Y. Identification of contaminant point source in surface waters based on backward location probability density function method. Adv Water Resour 2010;33(4):397-410. http://dx.doi.org/10.1016/j.advwatres.2010.01.004.

[15] Cherkassky V, Mulier F. Learning from data, concepts, theory and methods. New Jersey: Wiley; 2007

[16] China Statistical Yearbook. China environmental pollution and destruction accidents statistics over the years (2000-2008) 2009.

[17] Fedoseyev AI, Friedman MJ, Kansa EJ. Improved multiquadric method for elliptic partial differential equations via PDE collocation on the boundary. Comput Math Appl 2002;43:439-55. http://dx.doi.org/10.1016/S0898-1221(01)00297-8.

[18] Foley TA. Near optimal parameter selection for multiquadric interpolation. J Appl Sci Comput 1994;1(1):54-69.

[19] Franke C, Schaback R. Solving partial differential equations by collocation using radial basis functions. Appl Math Comput 1998;93(1):73-82. http://dx.doi.org/ 10.1016/S0096-3003(97)10104-7

[20] Franke R. Scattered data interpolation- tests of some methods. Math Comput 1982;38(157):181-200. http://dx.doi.org/10.1090/S0025-5718-1982-0637296-4.

[21] Hardy RL. Multiquadric equations of topography and other irregular surfaces. J Geophys Res 1971;76(8):1905-15. http://dx.doi.org/10.1029/JB076i008p01905.

[22] Hon YC, Cheung KF, Mao XZ, Kansa EJ. Multiquadric solution for shallow water equations. J Hydraul Eng 1999;125(5):524-33. http://dx.doi.org/10.1061/(ASCE) 0733-9429(1999)125:5(524).
[23] Huang CH, Li JX, Kim S. An inverse problem in estimating the strength of contaminant source for groundwater systems. Appl Math Model 2008;32(4):417-31. http://dx.doi.org/10.1016/j.apm.2006.12.009.

[24] Kansa EJ. Multiquadrics-a scattered data approximation scheme with applications to computational fluid dynamics-П Solutions to parabolic, hyperbolic and elliptic partial differential equations. Comput Math Appl 1990;19:147-61. http://dx.doi.org/10.1016/0898-1221(90)90271-K.

[25] Katopodes ND. Control of sudden releases in channel flow. Fluid Dyn Res 2009;41(6):1-24. http://dx.doi.org/10.1088/0169-5983/41/6/065002.

[26] Katopodes ND, Piasecki M. Site and size optimization of contaminant sources in surface water systems. J Environ Eng 1996;122(10):917-23. http://dx.doi.org/10. 1061/(ASCE)0733-9372(1996)122:10(917).

[27] Kee BBT, Liu GR, Lu C. A least-square radial point collocation method for adaptive analysis in linear elasticity. Eng Anal Boundary Elem 2008;32(6):440-60. http:// dx.doi.org/10.1016/j.enganabound.2007.11.011.

[28] Li Z, Mao XZ. Global space-time multiquadric method for inverse heat conduction problems. Int J Numer Meth Eng 2011;85(3):355-79. http://dx.doi.org/10.1002/ nme.2975.

[29] Li Z, Mao XZ. Global multiquadric collocation method for groundwater contaminant source identification. Environ Model Softw 2011;26:161-1621. http://dx.doi. org/10.1016/j.envsoft.2011.07.010.

[30] Madych WR. Miscellaneous error bounds for multiquadric and related interpolators. Comput Math Appl 1992;24(12):121-38. http://dx.doi.org/10.1016/ 0898-1221(92)90175- $\mathrm{H}$.

[31] Mao XZ, Li Z. Least square-based radial basis collocation method for solving inverse problems of Laplace equation from noisy data. Int J Numer Meth Eng 2010;84(1):1-26. http://dx.doi.org/10.1002/nme.2880.

[32] Michalak AM, Kitanidis PK. Estimation of historical groundwater contaminant distribution using the adjoint state method applied to geostatistical inverse modeling. Water Resour Res 2004;40(8):W08302. http://dx.doi.org/10.1029/ 2004WR003214.

[33] Piasecki M, Katopodes ND. Control of contaminant releases in rivers and estuaries. Part II: optimal design. J Hydraul Eng 1997;123(6):493-503. http://dx.doi.org/10. 1061/(ASCE)0733-9429(1997)123:6(493).

[34] Rippa S. An algorithm for selecting a good value for the parameter $\mathrm{c}$ in radial basis function interpolation. Adv Comput Math 1999;11:193-210. http://dx.doi.org/10. 1023/A:1018975909870

[35] Roque CMC, Ferreira AJM. Numerical experiments on optimal shape parameters for radial basis functions. Numer Meth Part Differ Equ 2010;26(3):675-89. http:// dx.doi.org/10.1002/num.20453.

[36] Shlomi S, Michalak AM. A geostatistical framework for incorporating transport information in estimating the distribution of a groundwater contaminant plume. Water Resour Res 2007;43(3):W03412. http://dx.doi.org/10.1029/ 2006 WR005121.

[37] Singh RM, Datta B, Jain A. Identification of unknown groundwater pollution sources using artificial neural networks. J Water Resour Plan Manage 2004;130(6):506-14. http://dx.doi.org/10.1061/(ASCE)0733-9496(2004)130: 6(506).

[38] Skaggs TH, Kabala ZJ. Limitations in recovering the history of a groundwater contaminant plume. J Contam Hydrol 1998;33:347-59. http://dx.doi.org/10.1016/ S0169-7722(98)00078-3.

[39] Srivastava D, Singh RM. Breakthrough curves characterization and identification of an unknown pollution source in groundwater system using an artificial neural network (ANN). Environ Forens 2014;15(2):175-89. http://dx.doi.org/10.1080/ 15275922.2014.890142.

[40] Sun AY, Painter SL, Wittmeyer GW. A robust approach for iterative contaminant source location and release history recovery. J Contam Hydrol 2006;88:181-96. http://dx.doi.org/10.1016/j.jconhyd.2006.06.006.

[41] Tsaia CH, Kolibala J, Li M. The golden section search algorithm for finding a good shape parameter for meshless collocation methods. Eng Anal Boundary Elem 2010;34(8):738-46. http://dx.doi.org/10.1016/j.enganabound.2010.03.003.

[42] Wu Z, Schaback R. Local error estimates for radial basis function interpolation of scattered data. IMA J Numer Anal 1993;13:13-27. http://dx.doi.org/10.1093/ imanum/13.1.13.

[43] Zhang B, Wang Q, Li S, et al. Simulation of water quality for Songhua river water pollution accident using a one-dimensional water quality simulation model based system dynamics. China Environ Sci 2007;27(6):811-15 (in Chinese). 